Copyright (C) 2016 IEEE. Personal use of this material is permitted. Permission from IEEE must be obtained for all other uses, in any current or future media, including reprinting/republishing this material for advertising or promotional purposes, creating new collective works, for resale or redistribution to servers or lists, or reuse of any copyrighted component of this work in other works. 


\title{
Array-Aided Multi-Frequency GNSS Ionospheric Sensing: Estimability and Precision Analysis
}

\author{
Amir Khodabandeh and Peter J. G. Teunissen, Senior Member, IEEE
}

\begin{abstract}
The dual-frequency Global Positioning System (GPS) has proven to be an effective means of measuring the Earth's ionosphere and its Total Electron Content (TEC). With the advent of multi-frequency signals from more Global Navigation Satellite Systems (GNSSs), the opportunity arises to construct many more ionosphere-sensing combinations of GNSS data. With such diversity, various estimable ionospheric delays with differing interpretations (and of different precision) can be formed. How such estimable ionospheric delays should be interpreted, and the extent to which they contribute to the precision with which the unbiased TEC can be estimated, are the topics of this contribution. Based on multi-frequency GNSS code-only, phase-only and phase- and code data, we derive the closed-form solutions of different types of ionospheric observables that each can serve as input of an externally provided ionospheric model for TEC determination. Within such a general least-squares framework, we generalize the widely-used Phase to Code Levelling (PCL) technique to its multi-frequency version. We also show that only certain specific linear combinations of the observables contribute to the TEC solutions. As a further improvement of the multi-frequency GNSS-derived TEC solution, we propose and study the usage of an array of GNSS antennas. Analytical solutions, supported by numerical examples, of this array-based concept are presented, together with a discussion on its relevance for TEC determination. This concerns the roles of time-averaging and time-differencing, of integer ambiguity resolution, and of the number of frequencies and number of array antennas in determining TEC.
\end{abstract}

\section{Index Terms}

Global Navigation Satellite Systems (GNSS), Total Electron Content (TEC), Phase to Code Levelling (PCL), Phase-driven and Code-driven estimability.

\section{INTRODUCTION}

G LOBAL Navigation Satellite Systems (GNSS) have proven to be an effective means of measuring the Earth's ionosphere and its Total Electron Content (TEC). The observed ionospheric delays on the carrier-phase and code (pseudo-range) signals are not only valuable sources for ionospheric studies [1]-[11], but also for analyzing and assessing their impact on other GNSS-derived parameters of importance for Earth science disciplines (e.g., positioning, deformation, troposphere) [12]-[18].

It is well-known that with GPS dual-frequency data, the geometry-free linear combinations for determining ionospheric delays are accompanied by additional unknown parameters, i.e. the carrier-phase ambiguities and/or the code biases [5]. Such GPS-derived ionospheric delays do therefore not represent the 'unbiased' TEC, but a combination of the TEC and the GPS instrumental biases. One can retrieve the unbiased TEC however, by taking recourse to an ionospheric model. The fact that the geometry-free combinations cannot unbiasedly determine the slant ionospheric delays without an ionospheric model, reveals the intrinsic lack of information content in the GNSS data. As a consequence of this lack of information, different estimable combinations can be formed that may serve as input of the ionospheric model.

With the advent of multi-frequency signals from more GNSSs, the opportunity arises to construct even more such ionospheresensing combinations of GNSS data. With such diversity, various estimable ionospheric delays with differing interpretations (and of different precision) can be formed. How such estimable ionospheric delays should be interpreted, and the extent to which they contribute to the precision with which the unbiased TEC can be estimated, are the topics of this contribution. Other GNSS ionospheric-sensing tools in the literature, like the radio occultation measurements [10], [19], are not considered in this contribution.

In order to properly interpret the lack of information content in the GNSS data, we apply $\mathcal{S}$-system theory [20], [21] to analyse the rank-deficient system of GNSS observation equations. By using a step-wise approach in making this system of equations full-rank, we show how different types of estimable ionospheric functions can be formed and how they are related. As a further improvement of the multi-frequency GNSS-derived TEC solution, we introduce and develop the array-aided concept of multi-frequency TEC determination. Analytical solutions of our array-aided concept are presented, together with a discussion on its relevance for TEC determination. This concerns the roles of time-averaging and time-differencing, of integer ambiguity resolution, and of the number of frequencies and number of array antennas in determining TEC.

A. Khodabandeh is with the GNSS Research Centre, Department of Spatial Sciences, Curtin University of Technology, Perth, Australia (email: amir.khodabandeh@curtin.edu.au).

P.J.G. Teunissen is with the GNSS Research Centre, Department of Spatial Sciences, Curtin University of Technology, Perth, Australia. He is also with the Department of Geoscience and Remote Sensing, Delft University of Technology, Delft, The Netherlands (e-mail: p.teunissen@curtin.edu.au).

Manuscript received ,; revised, 
This contribution is organized as follows. In Sect. II we first treat the rank-deficient GNSS observation equations in two different ways and express their full-rank models through two different presentations. Although each of these presentations leads to a different ionospheric observable, it is shown that they are re-parametrized versions of one another. Next to the estimability of the ionospheric observables, the closed-form expressions of their least-squares solutions are presented in Sect. III. These are followed by their corresponding (co)variance matrices. The solutions of the ionospheric observables over $k$ observational epochs are decomposed into their time-averaged and time-differenced components to better understand how the GNSS carrierphase and code measurements propagate into the solutions. We also generalize the well-known dual-frequency 'phase to code levelling' (PCL) procedure (see e.g. [4], [8], [22], [23]) to the multi-frequency case and show how it is linked to our general least-squares framework.

In Sect. IV we generalize the single-antenna solutions of the ionospheric observables to those that are obtained by an array of multiple antennas. The corresponding precision improvement is quantified and it is shown how the ionospheric observables have distinct responses to array ambiguity-resolution [24], [25]. The component of the ionospheric observables that takes an active role in determining the unbiased TEC solutions is discussed in Sect. V. By showing that only certain linear combinations of the observables contribute to the TEC solutions, we warn for the pitfall that exists when the quality of the TEC solutions is judged on the basis of the precision of the corresponding ionospheric observables. A summary with conclusions is finally provided in Sect. VI.

We make use of the following notation: The covariance and dispersion operators are denoted as $\mathrm{C}(.,$.$) and \mathrm{D}($.$) , respectively.$ Thus $\mathrm{C}(\boldsymbol{x}, \boldsymbol{x})=\mathrm{D}(\boldsymbol{x})$, with $\boldsymbol{x}$ being a random vector. The identity matrix of order $n$ is denoted as $\boldsymbol{I}_{n}$. The $n$-vector of ones (the summation vector) is denoted by $\boldsymbol{e}_{n}$. Wherever the subscript $n$ is omitted, the order of $\boldsymbol{I}$ and the size of the summation vector $\boldsymbol{e}$ are meant to be equal to the number of GNSS frequencies $f$. Thus $\boldsymbol{I}=\boldsymbol{I}_{f}$ and $\boldsymbol{e}=\boldsymbol{e}_{f}$. For the vectorial and multivariate representations, use is made of the matrix Kronecker product symbolized by $\otimes[26]$.

\section{IONOSPHERIC ESTIMABILITY VIA GNSS DATA}

\section{A. GNSS observation equations}

Consider the antenna $r$ tracking $f$-frequency GNSS data that are transmitted by the satellite $s$. Assuming a priori corrections such as the phase wind-up terms are applied, the corresponding observation equations read [27], [28]

$$
\begin{aligned}
& \phi_{r, j}^{s}(t)=-\mu_{j} i_{r}^{s}(t)+\rho_{r}^{s}(t)+a_{r, j}^{s} \\
& p_{r, j}^{s}(t)=+\mu_{j} i_{r}^{s}(t)+\rho_{r}^{s}(t)+d_{r, j}^{s}
\end{aligned}
$$

where $\phi_{r, j}^{s}$ and $p_{r, j}^{s}$ denote the phase and code observables on the frequency band $f_{j}(j=1, \ldots, f)$, respectively. The index $t$ refers to the epoch at which the observations are collected. The (first-order) slant ionospheric delay, experienced on the first frequency, is denoted by $i_{r}^{s}$. Thus the frequency-dependent coefficients are defined as the ratio $\mu_{j}=\left(f_{1}^{2} / f_{j}^{2}\right)$. The nondispersive delays, including the geometric range, clocks and the troposphere, are denoted by $\rho_{r}^{s}$. The real-valued ambiguity $a_{r, j}^{s}$ is expressed in units of range rather in cycles. The lumped term $d_{r, j}^{s}=d_{r, j}-d_{, j}^{s}$ contains the receiver and satellite code biases $d_{r, j}$ and $d_{, j}^{s}$, respectively. In this study, the ambiguities $a_{r, j}^{s}$ and code biases $d_{r, j}^{s}$ are assumed constant over time. That is why the epoch argument $t$ is omitted. The time-interval within which the ambiguities behave constant is the duration of a continuous satellite phase arc. In case of the code biases, their intra-day and daily changes have been investigated and reported, see e.g. [29], [30]. Under the nominal conditions, the code biases can be assumed stable during 1-3 days, see e.g. [3], [5], [8].

Introducing the multi-frequency vector notation $\boldsymbol{\phi}_{r}^{s}=\left[\phi_{r, 1}^{s}, \ldots, \phi_{r, f}^{s}\right]^{T}, \boldsymbol{p}_{r}^{s}=\left[p_{r, 1}^{s}, \ldots, p_{r, f}^{s}\right]^{T}, \boldsymbol{\mu}=\left[\mu_{1}, \ldots, \mu_{f}\right]^{T}, \boldsymbol{a}_{r}^{s}=$ $\left[a_{r, 1}^{s}, \ldots, a_{r, f}^{s}\right]^{T}$, and $\boldsymbol{d}_{r}^{s}=\left[d_{r, 1}^{s}, \ldots, d_{r, f}^{s}\right]^{T}$, the observation equations (1) can be expressed as

$$
\begin{aligned}
& \boldsymbol{\phi}_{r}^{s}(t)=-\boldsymbol{\mu} i_{r}^{s}(t)+\boldsymbol{e} \rho_{r}^{s}(t)+\boldsymbol{a}_{r}^{s} \\
& \boldsymbol{p}_{r}^{s}(t)=+\boldsymbol{\mu} i_{r}^{s}(t)+\boldsymbol{e} \rho_{r}^{s}(t)+\boldsymbol{d}_{r}^{s}
\end{aligned}
$$

In case one has no particular interest in the non-dispersive delays $\rho_{r}^{s}$, the geometry-free (GF) combinations are then often formed to eliminate the stated delays [3]-[5], [8], [9], [31]. The GF combinations are structured by differencing observables on two different frequencies, upon which only the slant ionospheric delays plus ambiguity/code biases do remain in the observation equations. Such combinations follow from (2) as

$$
\begin{array}{lll}
\text { Phase-based: } & -\boldsymbol{\mu}_{G F}^{T} \boldsymbol{\phi}_{r}^{s}(t)=i_{r}^{s}(t)-\boldsymbol{\mu}_{G F}^{T} \boldsymbol{a}_{r}^{s} \\
\text { Code-based: } & +\boldsymbol{\mu}_{G F}^{T} \boldsymbol{p}_{r}^{s}(t)=i_{r}^{s}(t)+\boldsymbol{\mu}_{G F}^{T} \boldsymbol{d}_{r}^{s}
\end{array}
$$

where

$$
\boldsymbol{\mu}_{G F}=\frac{1}{\mu_{2}-\mu_{1}}[-1,1,0, \ldots, 0]^{T}
$$

Note that here the combinations are made by the first two frequencies $j=1,2$, as it is the case with the GPS dual-frequency data. In case of multi-frequency data, one can also think of GF combinations of other than the first two frequencies. 
TABLE I

PHASE-DRIVEN ESTIMABLE FUNCTIONS FORMED BY LUMPING THEIR CORRESPONDING $\mathcal{S}$-BASIS PARAMETERS OF THE FULL-RANK MODEL (11).

\begin{tabular}{ll}
\hline Phase-driven ionospheric delays & $i_{r ; \phi}^{s}(t)=i_{r}^{s}(t)-\frac{1}{\mu_{12}}\left(a_{r, 2}^{s}-a_{r, 1}^{s}\right)$ \\
\hline Non-dispersive parameters & $\rho_{r ; \phi}^{s}(t)=\rho_{r}^{s}(t)+\frac{1}{\mu_{12}}\left(\mu_{2} a_{r, 1}^{s}-\mu_{1} a_{r, 2}^{s}\right)$ \\
\hline Estimable ambiguities & $a_{r, j ; \phi}^{s}=a_{r, j}^{s}+\frac{1}{\mu_{12}}\left[\mu_{2 j} a_{r, 1}^{s}-\mu_{1 j} a_{r, 2}^{s}\right] ; j>2$ \\
\hline Estimable code biases & $d_{r, j ; \phi}^{s}=d_{r, j}-d_{, j}^{s}+\frac{1}{\mu_{12}}\left[\left(\mu_{1}+\mu_{j}\right) a_{r, 2}^{s}-\left(\mu_{2}+\mu_{j}\right) a_{r, 1}^{s}\right]$ \\
\hline $\mathcal{S}$-basis parameters & $a_{r, 1}^{s}, a_{r, 2}^{s}(s=1, \ldots, m)$ \\
\hline & $(.)_{1 j}=(.)_{j}-(.)_{1}$
\end{tabular}

Great importance has been attached to the GF observables (3), even up to the point that they are sometimes referred to as the ionospheric observables [32]. With the sole use of the GF combinations, the slant ionospheric delays $i_{r}^{s}$ cannot however be separated from the ambiguity/code biases $\boldsymbol{a}_{r}^{s}$ and $\boldsymbol{d}_{r}^{s}$, due to their linear dependency. The GNSS observation equations (2), and in turn, the ionospheric observables (3) are thus not capable of determining these delays in an absolute sense. The stated linear dependency can be tackled though, at the expense of lumping the ambiguity- or the code-biases with the slant ionosphere.

In the following, we show the existence of further linear dependencies among the parameters. These linear dependencies can be taken care of by lumping a minimum set of parameters as the $\mathcal{S}$ ingularity-basis ( $\mathcal{S}$-basis) [21], imposing constraints on the rank-deficient model (2). Upon lumping the $\mathcal{S}$-basis parameters, the model becomes full-rank, thereby making a set of combined parameters estimable. Various estimable parameters can be formed though, depending on the choice of the $\mathcal{S}$-basis parameters. The following subsections present two examples of such $\mathcal{S}$-bases, leading to different estimability for the slant ionospheric delays $i_{r}^{s}$ (see Figure 1).

\section{B. Phase-driven estimability}

While the ionospheric observables (3) show that the slant ionospheric measurement can be carried out either by the code data or by the precise carrier-phase data [4], there are some studies that prefer the phase-based ionospheric observables so as to avoid the code mis-modeled effects such as multipath, see e.g. [8] or [9]. In this subsection, we therefore base the estimability of the GNSS parameters, given in (2), on the phase-only data to see how such estimable slant ionospheric delays are interpreted. As such an estimability analysis is driven by the carrier-phase data, the corresponding estimable parameters are hereafter referred to as the phase-driven parameters.

Ambiguity decomposition. To make the phase observation equations, given in (2), full-rank, we first make use of the multifrequency matrix identity [33]

$$
\boldsymbol{I}=\boldsymbol{e} \boldsymbol{\mu}_{I F}^{T}+\boldsymbol{\mu} \boldsymbol{\mu}_{G F}^{T}+\boldsymbol{E} \boldsymbol{E}^{-}
$$

with

$$
\begin{aligned}
\boldsymbol{\mu}_{I F} & =\frac{1}{\mu_{2}-\mu_{1}}\left[\mu_{2},-\mu_{1}, 0, \ldots, 0\right]^{T} \\
\boldsymbol{E}^{-} & =\boldsymbol{E}^{T}\left(\boldsymbol{I}-\boldsymbol{e} \boldsymbol{\mu}_{I F}^{T}-\boldsymbol{\mu} \boldsymbol{\mu}_{G F}^{T}\right)
\end{aligned}
$$

in which the $f \times(f-2)$ matrix $\boldsymbol{E}$ is structured by eliminating the first two columns of the identity matrix $\boldsymbol{I}$. The subscript 'IF' stands for the 'ionosphere-free' combinations.

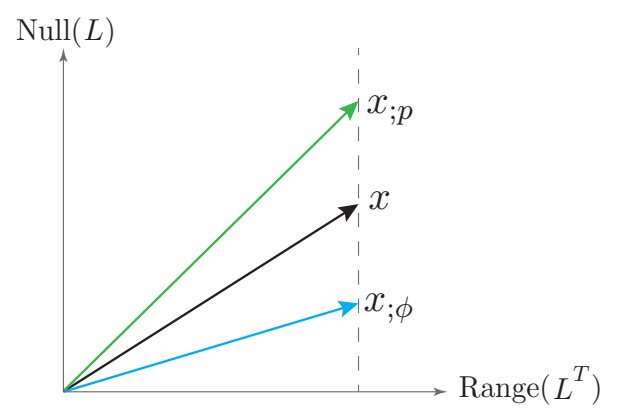

Fig. 1. Singularity-basis mechanism linking the 'absolute' unknown parameters $x$ to their 'estimable' counterparts $x_{; \phi}$ and $x_{;} p$. The rank-deficient design matrix $L$ contains the known coefficients in the linear model (2), while $x$ contains the unknown parameters $i_{r}^{s}(t), \rho_{r}^{s}(t), \boldsymbol{a}_{r}^{s}$ and $\boldsymbol{d}_{r}^{s}$. By choosing two different $\mathcal{S}$-bases, the absolute parameters $x$ are mapped to their phase-driven (blue) and code-driven (green) estimable counterparts $x_{; \phi}$ and $x ; p$, respectively. They are mapped along the direction of the null-space of $L\left(\operatorname{Null}(L):\right.$ dashed line) that is orthogonal to the range-space of the transpose of $L$, i.e. Range $\left(L^{T}\right)$. 
Application of (5) to the ambiguities $\boldsymbol{a}_{r}^{s}$ gives

$$
\boldsymbol{a}_{r}^{s}=\boldsymbol{e}\left(\boldsymbol{\mu}_{I F}^{T} \boldsymbol{a}_{r}^{s}\right)+\boldsymbol{\mu}\left(\boldsymbol{\mu}_{G F}^{T} \boldsymbol{a}_{r}^{s}\right)+\boldsymbol{E}\left(\boldsymbol{E}^{-} \boldsymbol{a}_{r}^{s}\right)
$$

The above ambiguity-decomposition shows that there exist linear dependencies between $\boldsymbol{\mu}_{I F}^{T} \boldsymbol{a}_{r}^{s}$ and $\rho_{r}^{s}$ in (2), and similarly between $\boldsymbol{\mu}_{G F}^{T} \boldsymbol{a}_{r}^{s}$ and $i_{r}^{s}$, as they have the common coefficients $\boldsymbol{e}$ and $\boldsymbol{\mu}$, respectively. Substituting (7) into the phase observation equations of (2), together with lumping $\boldsymbol{\mu}_{I F}^{T} \boldsymbol{a}_{r}^{s}$ and $\boldsymbol{\mu}_{G F}^{T} \boldsymbol{a}_{r}^{s}$, gives therefore the full-rank phase-only model

$$
\boldsymbol{\phi}_{r}^{s}(t)=-\boldsymbol{\mu} i_{r ; \phi}^{s}(t)+\boldsymbol{e} \rho_{r ; \phi}^{s}(t)+\boldsymbol{E} \boldsymbol{a}_{r ; \phi}^{s}
$$

in which the phase-driven estimable parameters, denoted by $(.)_{; \phi}$, have the following interpretation

$$
\begin{aligned}
& i_{r ; \phi}^{s}(t)=i_{r}^{s}(t)-\boldsymbol{\mu}_{G F}^{T} \boldsymbol{a}_{r}^{s} \\
& \rho_{r ; \phi}^{s}(t)=\rho_{r}^{s}(t)+\boldsymbol{\mu}_{I F}^{T} \boldsymbol{a}_{r}^{s} \\
& \boldsymbol{a}_{r ; \phi}^{s}=\boldsymbol{E}^{-} \boldsymbol{a}_{r}^{s}
\end{aligned}
$$

The following important remarks can now be made with respect to the full-rank model (8). First, the phase-driven estimable ionospheric delay $i_{r ; \phi}^{s}$ is so-called unlevelled, since it is biased by the geometry-free ambiguity combination $\boldsymbol{\mu}_{G F}^{T} \boldsymbol{a}_{r}^{s}$. Provided that no slip occurs during an arc of one tracked satellite, one can initially assume that this bias remains constant over time. The unbiased slant TEC can therefore be retrieved by any estimate of the phase-driven ionospheric delay $i_{r ; \phi}^{s}$ through the so-called arc-by-arc calibration technique, see e.g. the contribution by [9].

Second, the phase-driven estimable ambiguities $\boldsymbol{a}_{r ; \phi}^{s}$ do only appear in the observation equations on the third frequency and beyond, i.e. $j>2$. The ambiguities on the first two frequencies, $j=1,2$, are namely not estimable as they are already chosen as the $\mathcal{S}$-basis and lumped with the remaining parameters. Note that this is due to the choice of $\mathcal{S}$-basis of the underlying model. If one were to choose another $\mathcal{S}$-basis (cf. the following subsection) and/or take a different underlying model, such as the geometry-based models [34], estimable ambiguities (of different interpretations) would be formed.

Inclusion of the code-data. Although not necessary, let us, for the sake of comparison, include the code observation equations in the full-rank model (8). From the first two expressions of (9), it follows that

$$
\begin{aligned}
i_{r}^{s}(t) & =i_{r ; \phi}^{s}(t)+\boldsymbol{\mu}_{G F}^{T} \boldsymbol{a}_{r}^{s} \\
\rho_{r}^{s}(t) & =\rho_{r ; \phi}^{s}(t)-\boldsymbol{\mu}_{I F}^{T} \boldsymbol{a}_{r}^{s}
\end{aligned}
$$

Substitution into the code observation equations of (2), together with the phase-only model (8), gives the phase-and-code full-rank model

$$
\begin{aligned}
& \phi_{r}^{s}(t)=-\boldsymbol{\mu} i_{r ; \phi}^{s}(t)+\boldsymbol{e} \rho_{r ; \phi}^{s}(t)+\boldsymbol{E} \boldsymbol{a}_{r ; \phi}^{s} \\
& \boldsymbol{p}_{r}^{s}(t)=+\boldsymbol{\mu} i_{r ; \phi}^{s}(t)+\boldsymbol{e} \rho_{r ; \phi}^{s}(t)+\boldsymbol{d}_{r ; \phi}^{s}
\end{aligned}
$$

where

$$
\boldsymbol{d}_{r ; \phi}^{s}=\boldsymbol{d}_{r}^{s}+\left(\boldsymbol{\mu} \boldsymbol{\mu}_{G F}^{T}-e \boldsymbol{\mu}_{I F}^{T}\right) \boldsymbol{a}_{r}^{s}
$$

Table I presents the phase-driven estimable parameters. Accordingly, the ambiguities on the first two frequencies (i.e. $\boldsymbol{a}_{r, 1}^{s}$ and $\boldsymbol{a}_{r, 2}^{s}$ ) are chosen as the $\mathcal{S}$-basis of the full-rank model (11).

\section{Code-driven estimability}

Biased by the ambiguities, the unlevelled ionospheric delays $i_{r ; \phi}^{s}$ can be leveled to its code-based counterpart through the widely-used technique of 'phase to code levelling' (PCL), see e.g., [4], [8], [22], [23]. We now base the estimability of the GNSS parameters, given in (2), on the code-only data to see how such estimable slant ionospheric delays are interpreted. Since such an estimability analysis is driven by the code data, the corresponding estimable parameters are hereafter referred to as the code-driven parameters.

Code-bias decomposition. Instead of the ambiguities, one may apply the multi-frequency matrix identity (5) to the code biases $\boldsymbol{d}_{r}^{s}$ (compare with 7)

$$
\boldsymbol{d}_{r}^{s}=\boldsymbol{e}\left(\boldsymbol{\mu}_{I F}^{T} \boldsymbol{d}_{r}^{s}\right)+\boldsymbol{\mu}\left(\boldsymbol{\mu}_{G F}^{T} \boldsymbol{d}_{r}^{s}\right)+\boldsymbol{E}\left(\boldsymbol{E}^{-} \boldsymbol{d}_{r}^{s}\right)
$$

Instead of $\boldsymbol{\mu}_{I F}^{T} \boldsymbol{a}_{r}^{s}$ and $\boldsymbol{\mu}_{G F}^{T} \boldsymbol{a}_{r}^{s}$, the ionosphere-free and geometry-free code biases $\boldsymbol{\mu}_{I F}^{T} \boldsymbol{d}_{r}^{s}$ and $\boldsymbol{\mu}_{G F}^{T} \boldsymbol{d}_{r}^{s}$ can therefore be, respectively, lumped with $\rho_{r}^{s}$ and $i_{r}^{s}$. Substitution of (13) into the code observation equations of (2) results in the following full-rank code-only model (compare with 8 )

$$
\boldsymbol{p}_{r}^{s}(t)=+\boldsymbol{\mu} i_{r ; p}^{s}(t)+\boldsymbol{e} \rho_{r ; p}^{s}(t)+\boldsymbol{E} \boldsymbol{d}_{r ; p}^{s}
$$


TABLE II

CODE-DRIVEN ESTIMABLE FUNCTIONS FORMED BY LUMPING THEIR CORRESPONDING $\mathcal{S}$-BASIS PARAMETERS OF THE FULL-RANK MODEL (16).

\begin{tabular}{ll}
\hline Code-driven ionospheric delays & $i_{r ; p}^{s}(t)=i_{r}^{s}(t)+\frac{1}{\mu_{12}}\left(d_{r, 2}^{s}-d_{r, 1}^{s}\right)$ \\
\hline Non-dispersive parameters & $\rho_{r ; p}^{s}(t)=\rho_{r}^{s}(t)+\frac{1}{\mu_{12}}\left(\mu_{2} d_{r, 1}^{s}-\mu_{1} d_{r, 2}^{s}\right)$ \\
\hline Estimable ambiguities & $a_{r, j ; p}^{s}=a_{r, j}^{s}+\frac{1}{\mu_{12}}\left[\left(\mu_{1}+\mu_{j}\right) d_{r, 2}^{s}-\left(\mu_{2}+\mu_{j}\right) d_{r, 1}^{s}\right]$ \\
\hline Estimable code biases & $d_{r, j ; p}^{s}=d_{r, j}^{s}+\frac{1}{\mu_{12}}\left[\mu_{2 j} d_{r, 1}^{s}-\mu_{1 j} d_{r, 2}^{s}\right] ; j>2$ \\
\hline $\mathcal{S}$-basis parameters & $d_{r, 1}^{s}, d_{r, 2}^{s}(s=1, \ldots, m)$ \\
\hline & $(.)_{1 j}=(.)_{j}-(.)_{1}$
\end{tabular}

in which the code-driven estimable parameters, denoted by $(.)_{; p}$, have the following interpretation

$$
\begin{aligned}
i_{r ; p}^{s}(t) & =i_{r}^{s}(t)+\boldsymbol{\mu}_{G F}^{T} \boldsymbol{d}_{r}^{s} \\
\rho_{r ; p}^{s}(t) & =\rho_{r}^{s}(t)+\boldsymbol{\mu}_{I F}^{T} \boldsymbol{d}_{r}^{s} \\
\boldsymbol{d}_{r ; p}^{s} & =\boldsymbol{E}^{-} \boldsymbol{d}_{r}^{s}
\end{aligned}
$$

Compare the above estimable parameters with those of (9). The role of $\mathcal{S}$-basis parameters $\boldsymbol{\mu}_{I F}^{T} \boldsymbol{a}_{r}^{s}$ and $\boldsymbol{\mu}_{G F}^{T} \boldsymbol{a}_{r}^{s}$ has now been taken by $\boldsymbol{\mu}_{I F}^{T} \boldsymbol{d}_{r}^{s}$ and $\boldsymbol{\mu}_{G F}^{T} \boldsymbol{d}_{r}^{s}$. Likewise, the code-driven estimable code-biases $\boldsymbol{d}_{r ; p}^{s}$ do only appear in the observation equations on the third frequency and beyond, i.e. $j>2$, since those on the first frequencies are already lumped with the remaining parameters.

The code-driven estimable ionospheric delay $i_{r ; p}^{s}$ is biased by the geometry-free code-bias combination $\boldsymbol{\mu}_{G F}^{T} \boldsymbol{d}_{r}^{s}$. This code bias combination is referred to as the 'differential code bias' (DCB) which is also known as the inter-frequency bias [5]. Note that the DCB $\boldsymbol{\mu}_{G F}^{T} \boldsymbol{d}_{r}^{s}$ is specified by its corresponding satellite. The technique, retrieving the slant TEC through the estimates of the code-driven ionospheric delay $i_{r ; p}^{s}$, is therefore known as the satellite-by-satellite calibration technique [9].

Inclusion of the phase-data. To see how the code-driven ambiguities are interpreted, we now include the phase observation equations in the full-rank model (14). Using the first two expressions of (15), the phase-and-code full-rank model follows analogous to (11) as

$$
\begin{aligned}
& \phi_{r}^{s}(t)=-\boldsymbol{\mu} i_{r ; p}^{s}(t)+\boldsymbol{e} \rho_{r ; p}^{s}(t)+\boldsymbol{a}_{r ; p}^{s} \\
& \boldsymbol{p}_{r}^{s}(t)=+\boldsymbol{\mu} i_{r ; p}^{s}(t)+\boldsymbol{e} \rho_{r ; p}^{s}(t)+\boldsymbol{E} \boldsymbol{d}_{r ; p}^{s}
\end{aligned}
$$

where

$$
\boldsymbol{a}_{r ; p}^{s}=\boldsymbol{a}_{r}^{s}+\left(\boldsymbol{\mu} \boldsymbol{\mu}_{G F}^{T}-\boldsymbol{e} \boldsymbol{\mu}_{I F}^{T}\right) \boldsymbol{d}_{r}^{s}
$$

The code-driven estimable parameters are summarized in Table II. Compare the code-driven $\mathcal{S}$-basis with its phase-driven counterpart in Table I. While the phase-driven estimable parameters follow by lumping the ambiguities on the first two frequencies (i.e. $\boldsymbol{a}_{r, 1}^{s}$ and $\boldsymbol{a}_{r, 2}^{s}$ ), those of the code-driven model are formed by choosing the code biases on the first two frequencies (i.e. $\boldsymbol{d}_{r, 1}^{s}$ and $\boldsymbol{d}_{r, 2}^{s}$ ) as the $\mathcal{S}$-basis.

\section{Phase- and code-driven estimability compared}

In the two previous subsections, the GNSS rank-deficient model (2) was treated in two different ways, giving rise to two different sets of estimable parameters. Indeed, one can choose another set of $\mathcal{S}$-basis parameters that differ from those given in Tables I and II. The structure of the associated full-rank model would then also become different from those of (11) and (16), where its estimable ionospheric parameter would not be identical to the phase-driven $i_{r ; \phi}^{s}$, nor to the code-driven $i_{r ; p}^{s}$. One should therefore not directly compare, for instance, the precision of such a newly-defined estimable ionospheric parameter with those of $i_{r ; \phi}^{s}$ and $i_{r ; p}^{s}$. Likewise, the precision of any estimator of $i_{r ; \phi}^{s}$ is not comparable to that of estimators of $i_{r ; p}^{s}$, as they indeed describe two different quantities, i.e. $i_{r ; \phi}^{s} \neq i_{r ; p}^{s}$. They can, however, be linked to one another, would one properly take the role of other estimable parameters into account. To see this, let us re-write the interpretation of the phase-driven ionospheric delay $i_{r ; \phi}^{s}$ as follows

$$
\begin{aligned}
i_{r ; \phi}^{s}(t) & =\left(i_{r}^{s}(t)+\boldsymbol{\mu}_{G F}^{T} \boldsymbol{d}_{r}^{s}\right)-\boldsymbol{\mu}_{G F}^{T}\left(\boldsymbol{a}_{r}^{s}+\boldsymbol{d}_{r}^{s}\right) \\
& =\left(i_{r}^{s}(t)+\boldsymbol{\mu}_{G F}^{T} \boldsymbol{d}_{r}^{s}\right)-\boldsymbol{\mu}_{G F}^{T}\left(\boldsymbol{a}_{r}^{s}+\left[\boldsymbol{\mu} \boldsymbol{\mu}_{G F}^{T}-e \boldsymbol{\mu}_{I F}^{T}\right] \boldsymbol{d}_{r}^{s}\right) \\
& =i_{r ; p}^{s}(t)-\boldsymbol{\mu}_{G F}^{T} \boldsymbol{a}_{r ; p}^{s}
\end{aligned}
$$

The first equality follows by adding and subtracting $\boldsymbol{\mu}_{G F}^{T} \boldsymbol{d}_{r}^{s}$ from the first expression of (9), while the second equality follows from the identities $\boldsymbol{\mu}_{G F}^{T} \boldsymbol{\mu}=1$ and $\boldsymbol{\mu}_{G F}^{T} \boldsymbol{e}=0$. The last equality follows, respectively, from the first expression of (15) and (17). The last equality shows that the phase-driven ionospheric delay $i_{r ; \phi}^{s}$ is linked to its code-driven counterpart $i_{r ; p}^{s}$ through 
TABLE III

THE ONE-TO-ONE TRANSFORMATION BETWEEN THE PHASE-DRIVEN AND CODE-DRIVEN ESTIMABLE PARAMETERS

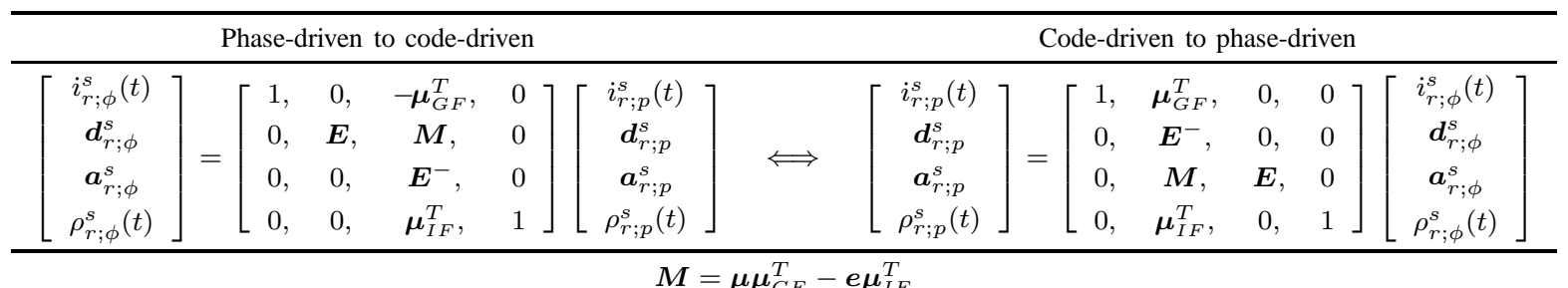

the code-driven ambiguity $\boldsymbol{a}_{r ; p}^{s}$. In an analogous way, the links between the other sets of estimable parameters can be made, the output of which results in a one-to-one relationship between the two sets of estimable parameters (see Table III).

Such a one-to-one transformation implies that the phase-and-code full-rank models (11) and (16) are re-parametrized versions of one another. This shows that both the models contain the same information, albeit expressed in two different presentations. Therefore, application of a rigorous least-squares adjustment to either (11) or (16) must give the same TEC results. We discuss TEC determination in Sect. V.

\section{PRECISION OF THE IONOSPHERIC OBSERVABLES}

\section{A. Ionospheric observables over $k$ epochs}

So far the estimability of the slant ionospheric delays, based on the GNSS data, is discussed. We now take this one step further by providing the solutions of the estimable slant ionospheric delays. Next to the solutions, their corresponding precision is also presented.

Given the interpretations attributed to the phase- and code-driven ionospheric delays $i_{r ; \phi}^{s}$ and $i_{r ; p}^{s}$ (cf. 9 and 15), our earlier ionospheric observables (3) already provide single-epoch solutions for $i_{r ; \phi}^{s}$ and $i_{r ; p}^{s}$ at epoch $t$, that is

$$
\begin{array}{ll}
\text { Phase-based: } & \hat{i}_{r ; \phi}^{s}(t)=-\boldsymbol{\mu}_{G F}^{T} \boldsymbol{\phi}_{r}^{s}(t) \\
\text { Code-based: } & \hat{i}_{r ; p}^{s}(t)=+\boldsymbol{\mu}_{G F}^{T} \boldsymbol{p}_{r}^{s}(t)
\end{array}
$$

We aim to generalize the above solutions to their multi-epoch versions over epochs $t=1, \ldots, k$. Since such solutions serve as input for TEC determination, they are hereafter referred to as the ionospheric observables over $k$ epochs.

Given an $m$ number of satellites $(s=1, \ldots, m)$, let us define the phase observation vector of antenna $r$ as $\phi_{r}=$ $\left[\phi_{r, 1}^{T}, \ldots, \phi_{r, f}^{T}\right]^{T}$ (of size $f$ times $m$ ), where $\phi_{r, j}=\left[\phi_{r, j}^{1}, \ldots, \phi_{r, j}^{m}\right]^{T}, j=1, \ldots, f$, with a likewise definition for the code observation vector $\boldsymbol{p}_{r}$. With these settings, the observation equations (2) take the following form

$$
\begin{aligned}
& \phi_{r}(t)=-\left(\boldsymbol{\mu} \otimes \boldsymbol{I}_{m}\right) \boldsymbol{i}_{r}(t)+\left(\boldsymbol{e} \otimes \boldsymbol{I}_{m}\right) \boldsymbol{\rho}_{r}(t)+\boldsymbol{a}_{r} \\
& \boldsymbol{p}_{r}(t)=+\left(\boldsymbol{\mu} \otimes \boldsymbol{I}_{m}\right) \boldsymbol{i}_{r}(t)+\left(\boldsymbol{e} \otimes \boldsymbol{I}_{m}\right) \boldsymbol{\rho}_{r}(t)+\boldsymbol{d}_{r}
\end{aligned}
$$

where

$$
\begin{aligned}
\boldsymbol{i}_{r} & =\left[i_{r}^{1}, \ldots, i_{r}^{m}\right]^{T} ; \boldsymbol{\rho}_{r}=\left[\rho_{r}^{1}, \ldots, \rho_{r}^{m}\right]^{T} \\
\boldsymbol{a}_{r} & =\left[\left[a_{r, 1}^{1}, \ldots, a_{r, 1}^{m}\right]^{T}, \ldots,\left[a_{r, f}^{1}, \ldots, a_{r, f}^{m}\right]^{T}\right]^{T} \\
\boldsymbol{d}_{r} & =\left[\left[d_{r, 1}^{1}, \ldots, d_{r, 1}^{m}\right]^{T}, \ldots,\left[d_{r, f}^{1}, \ldots, d_{r, f}^{m}\right]^{T}\right]^{T}
\end{aligned}
$$

To evaluate the precision of the ionospheric solution, one needs the precision of the GNSS data through their variance matrix. The variance matrix of the observables is assumed given as

$$
\mathrm{D}\left[\begin{array}{l}
\boldsymbol{\phi}_{r}(t) \\
\boldsymbol{p}_{r}(t)
\end{array}\right]=\sigma_{p}^{2}\left[\begin{array}{cc}
\epsilon \boldsymbol{I} & 0 \\
0 & \boldsymbol{I}
\end{array}\right] \otimes \boldsymbol{W}_{t}^{-1}
$$

with $\sigma_{p}$ being the zenith-referenced standard-deviation of the code observables. The scalar $\epsilon$ denotes the phase-to-code variance ratio. In most GNSS applications, the stated ratio is taken approximately as $\epsilon \approx 0.0001$, since the precision of the phase observables is almost two orders of magnitude better than its code counterpart. The elevation dependency of the satellites is captured by the $m \times m$ diagonal weight matrix $\boldsymbol{W}_{t}=\operatorname{diag}\left(w_{t}^{1}, \ldots, w_{t}^{m}\right)$ that changes in time as the satellites' elevation changes.

The system of equations (20) represents the vectorial form of the rank-deficient model (2). The vectorial forms of the full-rank models (11) and (16) follow in a similar way.

Definition 1: (Single-antenna full-rank models) The $m$-satellite, $f$-frequency vectorial forms of the phase-driven and codedriven full-rank models (11) and (16) at epoch $t$ are, respectively, defined as

$$
\begin{aligned}
& \phi_{r}(t)=-\left(\boldsymbol{\mu} \otimes \boldsymbol{I}_{m}\right) \boldsymbol{i}_{r ; \phi}(t)+\left(\boldsymbol{e} \otimes \boldsymbol{I}_{m}\right) \boldsymbol{\rho}_{r ; \phi}(t)+\left(\boldsymbol{E} \otimes \boldsymbol{I}_{m}\right) \boldsymbol{a}_{r ; \phi} \\
& \boldsymbol{p}_{r}(t)=+\left(\boldsymbol{\mu} \otimes \boldsymbol{I}_{m}\right) \boldsymbol{i}_{r ; \phi}(t)+\left(\boldsymbol{e} \otimes \boldsymbol{I}_{m}\right) \boldsymbol{\rho}_{r ; \phi}(t)+\boldsymbol{d}_{r ; \phi}
\end{aligned}
$$


and

$$
\begin{aligned}
& \phi_{r}(t)=-\left(\boldsymbol{\mu} \otimes \boldsymbol{I}_{m}\right) \boldsymbol{i}_{r ; p}(t)+\left(\boldsymbol{e} \otimes \boldsymbol{I}_{m}\right) \boldsymbol{\rho}_{r ; p}(t)+\boldsymbol{a}_{r ; p} \\
& \boldsymbol{p}_{r}(t)=+\left(\boldsymbol{\mu} \otimes \boldsymbol{I}_{m}\right) \boldsymbol{i}_{r ; p}(t)+\left(\boldsymbol{e} \otimes \boldsymbol{I}_{m}\right) \boldsymbol{\rho}_{r ; p}(t)+\left(\boldsymbol{E} \otimes \boldsymbol{I}_{m}\right) \boldsymbol{d}_{r ; p}
\end{aligned}
$$

for $t=1, \ldots, k$.

The task is to solve the $k$-epoch full-rank models (23) and (24) for $\boldsymbol{i}_{r ; \phi}$ and $\boldsymbol{i}_{r ; p}$, respectively. To facilitate our derivation, we first express the solutions in terms of their time-averaged and -differenced components.

Lemma 1: (Time-averaged/-differenced decomposition) Let $\hat{\boldsymbol{i}}_{r ; \diamond}(t)$ be the solution of the estimable ionospheric delay $\boldsymbol{i}_{r ; \diamond}(t)$ at epoch $t$ obtained by the full-rank models (23) and (24) based on $k$ epochs, with $\diamond=\{\phi, p\}$. Then, the solution can be expressed by its time-averaged and -differenced components as follows $(t \in\{1, \ldots, k\})$

$$
\hat{\boldsymbol{i}}_{r ; \diamond}(t)=\hat{\boldsymbol{i}}_{r ; \diamond}(\bar{t})+\left\{\hat{\boldsymbol{i}}_{r ; \diamond}(1 t)-\hat{\boldsymbol{i}}_{r ; \diamond}(1 \bar{t})\right\}
$$

with

$$
\hat{\boldsymbol{i}}_{r ; \diamond}(1 \bar{t})=\frac{1}{k} \overline{\boldsymbol{W}}^{-1} \sum_{t=1}^{k} \boldsymbol{W}_{t} \hat{\boldsymbol{i}}_{r ; \diamond}(1 t)
$$

The time-averaging and time-differencing operators are, respectively, defined as

$$
(.)(\bar{t})=\frac{1}{k} \overline{\boldsymbol{W}}^{-1} \sum_{t=1}^{k} \boldsymbol{W}_{t}(.)(t), \quad(.)(1 t)=(.)(t)-(.)(1)
$$

where $\overline{\boldsymbol{W}}=(1 / k) \sum_{t=1}^{k} \boldsymbol{W}_{t}$.

Proof: follows by expanding the definitions (27), together with the substitution of (26) into (25).

According to the above lemma, one can solve the time-averaged and-differenced versions of (23) and (24) for $\boldsymbol{i}_{r ; \diamond}(\bar{t})$ and $\boldsymbol{i}_{r ; \diamond}(1 t)$, respectively, where $\diamond=\{\phi, p\}$. Once the solutions $\hat{\boldsymbol{i}}_{r ; \diamond}(\bar{t})$ and $\hat{\boldsymbol{i}}_{r ; \diamond}(1 t)$ are obtained, the final solution $\hat{\boldsymbol{i}}_{r ; \diamond}(t)$ would then follow as their linear function given by (25). This appears to be appealing, since both the solutions $\hat{\boldsymbol{i}}_{r ; \diamond}(\bar{t})$ and $\hat{\boldsymbol{i}}_{r ; \diamond}(1 t)$ can be shown to be uncorrelated, thereby acting as the independent building blocks of $\hat{\boldsymbol{i}}_{r ; \diamond}(t)$. The $k$-epoch solutions of the time-averaged and -differenced components are presented below.

Theorem 1: (Ionospheric observables: single-antenna) With reference to the full-rank models (23) and (24), the time-averaged solution of $\boldsymbol{i}_{r ; \diamond}(\bar{t})$ and its variance matrix are given as

$$
\hat{\boldsymbol{i}}_{r ; \diamond}(\bar{t})=\left\{\begin{array}{rr}
-\left(\boldsymbol{\mu}_{G F}^{T} \otimes \boldsymbol{I}_{m}\right) \boldsymbol{\phi}_{r}(\bar{t}), & \diamond=\phi \\
+\left(\boldsymbol{\mu}_{G F}^{T} \otimes \boldsymbol{I}_{m}\right) \boldsymbol{p}_{r}(\bar{t}), & \diamond=p
\end{array}\right.
$$

and

$$
\mathrm{D}\left(\hat{\boldsymbol{i}}_{r ; \diamond}(\bar{t})\right)=\left(\frac{2 \sigma_{\diamond}^{2}}{k \mu_{12}^{2}}\right) \overline{\boldsymbol{W}}^{-1} ; \quad \diamond=\{\phi, p\}
$$

with $\mu_{12}=\mu_{2}-\mu_{1}$ and $\sigma_{\phi}^{2}=\epsilon \sigma_{p}^{2}$.

While the time-differenced solutions of $\boldsymbol{i}_{r ; \diamond}(1 t)$ and their (co)variance matrices are given as $(\diamond=\{\phi, p\})$

$$
\begin{aligned}
\hat{\boldsymbol{i}}_{r ; \diamond}(1 t)=\frac{1}{f \gamma}\left\{2 \epsilon\left(\boldsymbol{\mu}^{T} \otimes \boldsymbol{I}_{m}\right)\left[\boldsymbol{p}_{r}(1 t)-\boldsymbol{\phi}_{r}(1 t)\right]-\right. \\
\left.(1-\epsilon)\left([\boldsymbol{\mu}-\bar{\mu} \boldsymbol{e}]^{T} \otimes \boldsymbol{I}_{m}\right)\left[\epsilon \boldsymbol{p}_{r}(1 t)+\boldsymbol{\phi}_{r}(1 t)\right]\right\}
\end{aligned}
$$

and

$$
\mathrm{C}\left(\hat{\boldsymbol{i}}_{r ; \diamond}(1 t), \hat{\boldsymbol{i}}_{r ; \diamond}(1 l)\right)=\frac{\sigma_{\phi}^{2}}{f \gamma}(1+\epsilon)\left(\boldsymbol{W}_{1}^{-1}+\delta_{t l} \boldsymbol{W}_{t}^{-1}\right)
$$

with $\bar{\mu}=(1 / f) \sum_{j=1}^{f} \mu_{j}, \sigma_{\mu}^{2}=(1 / f) \sum_{j=1}^{f}\left(\mu_{j}-\bar{\mu}\right)^{2}$, and $\gamma=(1+\epsilon)^{2} \sigma_{\mu}^{2}+4 \epsilon \bar{\mu}^{2}$. The delta Kronecker $\delta_{t l}$ is defined as

$$
\delta_{t l}= \begin{cases}1, & t=l \\ 0, & t \neq l\end{cases}
$$

Proof: see Appendix.

Two important remarks should be made with respect to the outcomes of Theorem 1. First, we highlight the difference between the time-averaged solutions of the phase- and code-driven ionospheric delays, i.e. $\hat{\boldsymbol{i}}_{r ; \phi}(\bar{t}) \neq \hat{\boldsymbol{i}}_{r ; p}(\bar{t})$. The solution of $\boldsymbol{i}_{r ; \phi}(\bar{t})$ is fully driven by the phase-only data, whereas that of $\boldsymbol{i}_{r ; p}(\bar{t})$ is fully driven by the code-only data. This is also the case with their precision, as their variance matrices are related by

$$
\mathrm{D}\left(\hat{\boldsymbol{i}}_{r ; \phi}(\bar{t})\right)=\epsilon \mathrm{D}\left(\hat{\boldsymbol{i}}_{r ; p}(\bar{t})\right)
$$



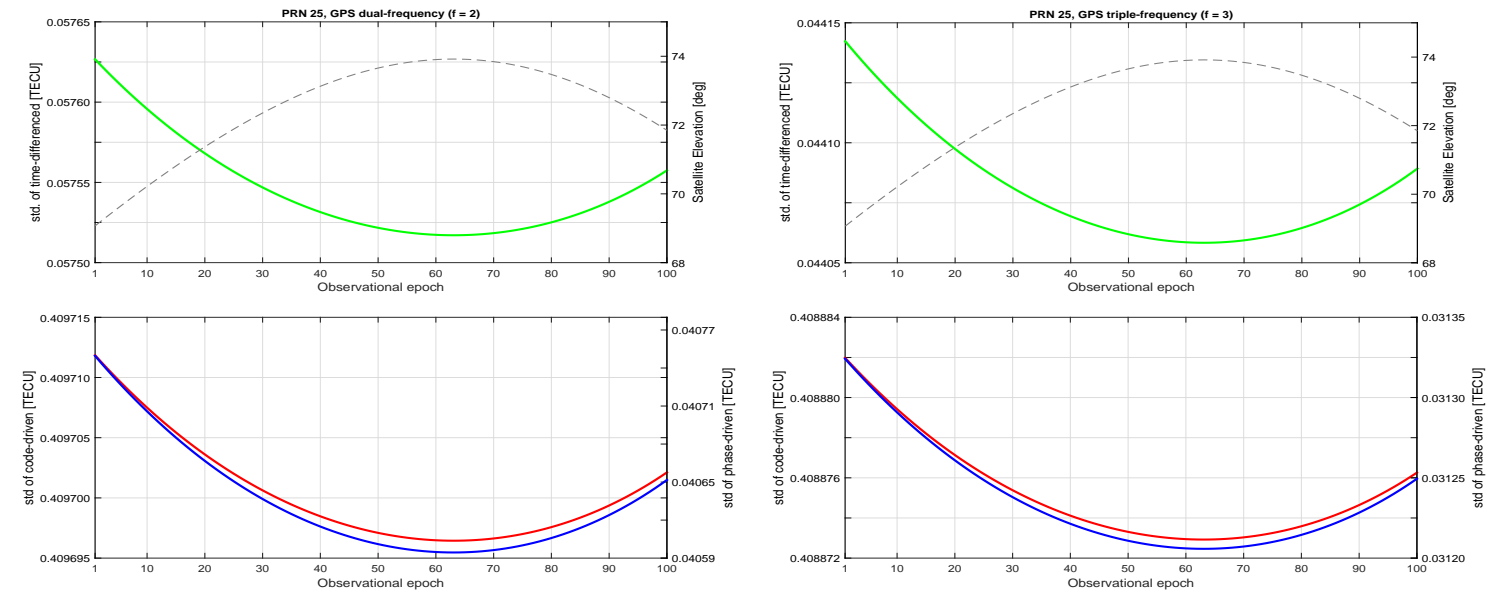

Fig. 2. Standard-deviation [TECU] of the time-differenced slant ionospheric solution (green lines) compared to those of the code-driven (red lines) and phase-driven (blue lines) solutions over epochs $t=1, \ldots, k(k=100)$. Left: PRN 25, GPS L1, L2 scenario ( $f=2)$; Right: PRN 25, GPS L1, L2, L5 scenario $(f=3)$. The values follow by setting $\sigma_{p}=30[\mathrm{~cm}] \approx 1.85$ [TECU], $\epsilon=0.0001$. The satellite elevation $\theta_{t}^{s}$ [deg] is depicted in grey dashed lines, where use is made of the exponential elevation weights $w_{t}^{s}=\left[1+10 \exp \left(-\theta_{t}^{s} / 10\right)\right]^{-2}$ [35]. The standard-deviations of the time-averaged solutions (code-driven: 0.40 [TECU], phase-driven: 0.04 [TECU]) dominate those of the ionospheric solutions.

The root of these differences lies in the different interpretations assigned to the estimable ionospheric parameters. The phasedriven ionospheric delay $\boldsymbol{i}_{r ; \phi}(\bar{t})$ is biased by the ambiguities, while the code-driven ionospheric delay $\boldsymbol{i}_{r ; p}(\bar{t})$ is biased by the code-biases (cf. 9 and 15). It is therefore evident that neither the least-squares solutions of two different quantities nor their precision are the same.

Second, we highlight the equality of the time-differenced solutions of the phase- and code-driven ionospheric delays, i.e. $\hat{\boldsymbol{i}}_{r ; \phi}(1 t)=\hat{\boldsymbol{i}}_{r ; p}(1 t)$. This is also what one would expect, since the stated ambiguity- and code-biases are eliminated from the estimable ionospheric delays, once the time-differencing is applied. The quantities $\boldsymbol{i}_{r ; \phi}(1 t)$ and $\boldsymbol{i}_{r ; p}(1 t)$ do in fact describe the same thing, namely, the time-differenced component of the unbiased ionospheric delays, that is

$$
\boldsymbol{i}_{r ; \phi}(1 t)=\boldsymbol{i}_{r ; p}(1 t)=\boldsymbol{i}_{r}(t)-\boldsymbol{i}_{r}(1)
$$

Their corresponding solutions should therefore be the same as well.

In the following, a few applications of Theorem 1 are presented, and the link to the well-known PCL procedure is also established.

\section{B. Slant ionospheric precision}

As an important application of Theorem 1, one can evaluate the precision of the slant ionospheric solutions $\hat{\boldsymbol{i}}_{r ; \phi}(t)$ and $\hat{\boldsymbol{i}}_{r ; p}(t)$ that is presented below.

Corollary 1: (Precision of the ionospheric observables: single-antenna) With reference to the full-rank models (23) and (24), the (co)variance matrices of the phase-driven and code-driven ionospheric observables are given by $(\diamond=\{\phi, p\})$

$$
\mathrm{C}\left(\hat{\boldsymbol{i}}_{r ; \diamond}(t), \hat{\boldsymbol{i}}_{r ; \diamond}(l)\right)=\left(\frac{2 \sigma_{\diamond}^{2}}{k \mu_{12}^{2}}\right) \overline{\boldsymbol{W}}^{-1}+\frac{\sigma_{\phi}^{2}}{f \gamma}(1+\epsilon)\left(\delta_{t l} \boldsymbol{W}_{t}^{-1}-\frac{1}{k} \overline{\boldsymbol{W}}^{-1}\right)
$$

Proof: Follows by an application of the variance propagation law to (25), together with the equalities (29) and (31). The above corollary shows that the precision of the phase-driven ionospheric observables $\hat{\boldsymbol{i}}_{r ; \phi}$ is governed by the precision of the carrier-phase data (i.e. $\sigma_{\phi}^{2}$ ), whereas that of the code-driven ionospheric observables $\hat{\boldsymbol{i}}_{r ; p}$ is dominated by the poor precision of the code data (i.e. $\sigma_{p}^{2}$ ). Note also that the difference in their precision is only due to the difference in the precision of their time-averaged components (cf. 29).

Figure 2 shows values of the standard-deviation of the solutions $\hat{\boldsymbol{i}}_{r ; \diamond}(t)(\diamond=\{\phi, p\})$ over epochs $t=1, \ldots, k(k=100)$. The standard-deviation changes as the elevation of the satellite changes. The higher the elevation, the smaller the standard-deviation becomes. The significant difference between the standard-deviation of the code-driven solutions $\hat{\boldsymbol{i}}_{r ; p}(t)$ (red lines) and that of their time-difference $\hat{\boldsymbol{i}}_{r ; p}(1 t)$ (green lines) is remarkable. The standard-deviation of $\hat{\boldsymbol{i}}_{r ; p}(t)$ is almost 10 times larger than that of $\hat{\boldsymbol{i}}_{r ; p}(1 t)$, as it is dominated by the code-based precision of the time-averaged solution $\hat{\boldsymbol{i}}_{r ; p}(\bar{t})$ (around 0.4 [TECU]). Note also that switching from the dual-frequency setup (left-panel) to the triple-frequency setup (right-panel) does not improve the precision of $\hat{\boldsymbol{i}}_{r ; p}(t)$ by much. In contrast to the code-driven solution $\hat{\boldsymbol{i}}_{r ; p}(t)$, the precision of the phase-driven solution $\hat{\boldsymbol{i}}_{r ; \phi}(t)$ (blue lines) is almost at the same level as that of their time-difference $\hat{\boldsymbol{i}}_{r ; \phi}(1 t)=\hat{\boldsymbol{i}}_{r ; p}(1 t)$, i.e. 10 times better than the precision of $\hat{\boldsymbol{i}}_{r ; p}(t)$. Would this mean that the phase-driven ionospheric solutions are preferred over their code-driven counterparts? Is the contribution of the additional frequency to TEC determination also marginal? We come back to these when we show how each of the solutions contributes to TEC determination (cf. Sect. V). 


\section{Phase-only solution}

The (co)variance matrices, given in (31), show that the time-differenced component solution $\hat{\boldsymbol{i}}_{r ; \diamond}(1 t)$ (with $\left.\diamond=\{\phi, p\}\right)$ is driven by the precision of the phase data, i.e. $\sigma_{\phi}^{2}=\epsilon \sigma_{p}^{2}$, where $\sigma_{\phi}$ denotes the zenith-referenced standard-deviation of the phase observables. One may then wonder as to whether it is possible to find a solution for $\boldsymbol{i}_{r ; \diamond}(1 t)$ that is obtained by the phase-data only. The answer is affirmative. Such a solution follows by zero-weighting the contribution of the code observables, i.e. by setting $\sigma_{p} \rightarrow \infty$ or equivalently $\epsilon \rightarrow 0$.

Corollary 2: (Phase-only time-differenced solution) The phase-only solutions of the time-differenced ionospheric parameters $\boldsymbol{i}_{r ; \phi}(1 t)$ and $\boldsymbol{i}_{r ; p}(1 t)$, together with their (co)variance matrices, are given as follows

$$
\hat{\boldsymbol{i}}_{r ; \diamond}(1 t)=\frac{-1}{f \sigma_{\mu}^{2}}\left([\boldsymbol{\mu}-\bar{\mu} \boldsymbol{e}]^{T} \otimes \boldsymbol{I}_{m}\right) \phi_{r}(1 t) ; \diamond=\{\phi, p\}
$$

and

$$
\mathrm{C}\left(\hat{\boldsymbol{i}}_{r ; \diamond}(1 t), \hat{\boldsymbol{i}}_{r ; \diamond}(1 l)\right)=\frac{\sigma_{\phi}^{2}}{f \sigma_{\mu}^{2}}\left(\boldsymbol{W}_{1}^{-1}+\delta_{t l} \boldsymbol{W}_{t}^{-1}\right)
$$

Proof: Follows by setting $\epsilon \rightarrow 0$ and $\left(\epsilon \sigma_{p}^{2}\right) \rightarrow \sigma_{\phi}^{2}$ in (30) and (31).

The phase-only solution of (36) is of particular importance, since it is exempted from any code mis-modeled effects such as multipath. Note that the structure of the phase-only solution (36) is simplified in the dual-frequency setup $(f=2)$. In that case, the solution becomes the straightforward GF combination of the phase observables, i.e.

$$
\hat{\boldsymbol{i}}_{r ; \diamond}(1 t)=-\left(\boldsymbol{\mu}_{G F}^{T} \otimes \boldsymbol{I}_{m}\right) \boldsymbol{\phi}_{r}(1 t) ; \diamond=\{\phi, p\}
$$

since

$$
\frac{1}{f \sigma_{\mu}^{2}}[\boldsymbol{\mu}-\bar{\mu} \boldsymbol{e}] \rightarrow \boldsymbol{\mu}_{G F}, \quad \text { when } \quad f=2
$$

\section{Relation to the PCL procedure}

Theorem 1 outlines steps towards computing the multi-epoch slant ionospheric solutions. Given the observables $\phi_{r}(t)$ and $\boldsymbol{p}_{r}(t)(t=1, \ldots, k)$, one can compute the components $\hat{\boldsymbol{i}}_{r ; \diamond}(\bar{t})$ and $\hat{\boldsymbol{i}}_{r ; \diamond}(1 t)$, respectively, through (28) and (30). Substitution into (25) gives therefore the least-squares solution for the estimable ionospheric delays $\boldsymbol{i}_{r ; \phi}(t)$ and $\boldsymbol{i}_{r ; p}(t)$. Through these steps, no mention of the widely-used 'phase to code levelling' (PCL) procedure has been made. Playing a prominent role in obtaining the slant ionospheric solutions however, the absence of the PCL procedure appears to be quite surprising. One would therefore expect that the PCL solution must fit into the general least-squares framework presented in Theorem 1. To address this, we make a start by the vectorial representation of (18) as

$$
\boldsymbol{i}_{r ; p}(t)=\boldsymbol{i}_{r ; \phi}(t)+\left(\boldsymbol{\mu}_{G F}^{T} \otimes \boldsymbol{I}_{m}\right) \boldsymbol{a}_{r ; p}
$$

We now follow [8, Sect. 2] to obtain the PCL solution. The phase-based single-epoch solution for $\boldsymbol{i}_{r ; \phi}(t)$ is given by (cf. 19)

$$
\hat{\boldsymbol{i}}_{r ; \phi}(t)=-\left(\boldsymbol{\mu}_{G F}^{T} \otimes \boldsymbol{I}_{m}\right) \phi_{r}(t)
$$

As the above phase-driven ionospheric solution is biased by the ambiguities, it is referred to as the 'unleveled' solution. This solution is leveled to the code observables, would the unknown ambiguity-bias $\boldsymbol{a}_{r ; p}$ be estimated. The ambiguity-bias is estimated by the time-average of the phase-plus-code observations

$$
\left(\boldsymbol{\mu}_{G F}^{T} \otimes \boldsymbol{I}_{m}\right) \hat{\boldsymbol{a}}_{r ; p}=\left(\boldsymbol{\mu}_{G F}^{T} \otimes \boldsymbol{I}_{m}\right)\left(\boldsymbol{\phi}_{r}(\bar{t})+\boldsymbol{p}_{r}(\bar{t})\right)
$$

where the averaging takes its simplest form by assuming no elevation-weighting, i.e. $\boldsymbol{W}_{t}=\boldsymbol{I}_{m}(t=1, \ldots, k)$. According to (40), the PCL solution follows by adding the ambiguity solution (42) to the unlevelled solution (41). Doing so, the PCL solution follows as

$$
\begin{aligned}
\hat{\boldsymbol{i}}_{r ; p}(t) & =\hat{\boldsymbol{i}}_{r ; \phi}(t)+\left(\boldsymbol{\mu}_{G F}^{T} \otimes \boldsymbol{I}_{m}\right) \hat{\boldsymbol{a}}_{r ; p} \\
& =\left(\boldsymbol{\mu}_{G F}^{T} \otimes \boldsymbol{I}_{m}\right)\left\{\boldsymbol{p}_{r}(\bar{t})+\left[\left(-\boldsymbol{\phi}_{r}(1 t)\right)-\frac{1}{k} \sum_{l=1}^{k}\left(-\boldsymbol{\phi}_{r}(1 l)\right)\right]\right\}
\end{aligned}
$$

Compare the PCL solution (43) with (25). Both are identical in structure. In fact, the PCL solution (43) follows from (25) by setting

$$
\diamond \rightarrow p, \quad, \boldsymbol{W}_{t} \rightarrow \boldsymbol{I}_{m}, \quad \hat{\boldsymbol{i}}_{r ; p}(\bar{t}) \rightarrow\left(\boldsymbol{\mu}_{G F}^{T} \otimes \boldsymbol{I}_{m}\right) \boldsymbol{p}_{r}(\bar{t})
$$

and

$$
\hat{\boldsymbol{i}}_{r ; p}(1 t) \rightarrow-\left(\boldsymbol{\mu}_{G F}^{T} \otimes \boldsymbol{I}_{m}\right) \phi_{r}(1 t)
$$

But this is the solution that is obtained by the phase-only time-differenced component (38). We therefore arrive at the following corollary. 


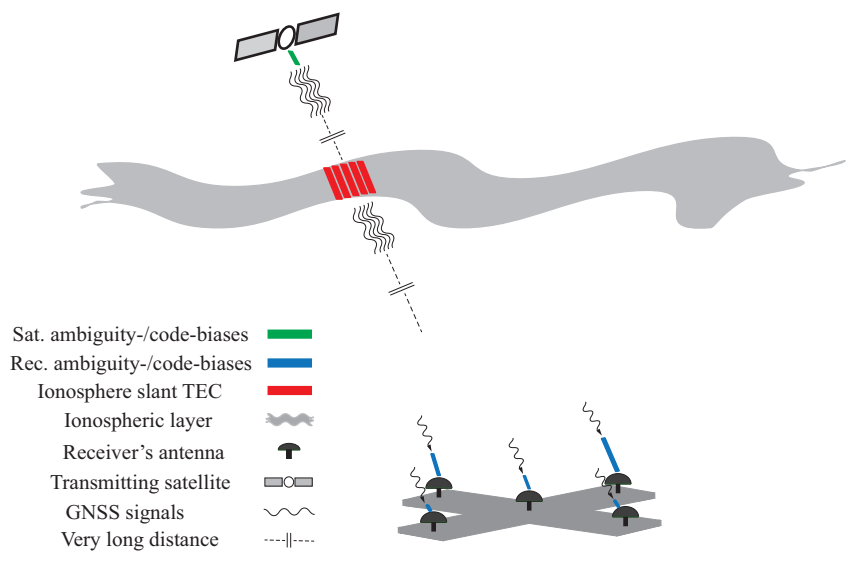

Fig. 3. Schematic illustration of the array-aided GNSS ionospheric sensing. The distance between the antennas are short enough so that almost the same ionospheric delays, of each satellite, are experienced by all the antennas.

Corollary 3: (PCL solution) The 'phase to code levelling' (PCL) solution of the estimable ionospheric delay $\boldsymbol{i}_{r ; p}(t)$ follows as a special case of the general solution (25), if

1) The data are restricted to dual-frequency $(f=2)$.

2) The time-differenced solution $\hat{\boldsymbol{i}}_{r ; p}(1 t)$ is obtained by the phase-only data.

3) No elevation weighting is applied, i.e. $\boldsymbol{W}_{t}=\boldsymbol{I}_{m}$.

Therefore, the general solution (25) encompasses the PCL solution as a special case. We remark that there are some studies applying the PCL procedure with an elevation weighting strategy, see e.g. [23]. In such cases, the last condition of Corollary 3 becomes obsolete. With the outcomes of Corollary 3, we indeed generalize the PCL technique to its multi-frequency version, i.e. when $f>2$. Accordingly, the multi-frequency PCL solution is structured by the phase-only time-differenced component $\hat{\boldsymbol{i}}_{r ; p}(1 t)=\hat{\boldsymbol{i}}_{r ; \phi}(1 t)$ and the code-driven time-averaged component $\hat{\boldsymbol{i}}_{r ; p}(\bar{t})$.

\section{THE ARRAY-AIDED CONCEPT}

\section{A. Array-aided ionospheric observables}

So far we restricted our ionospheric precision analysis to the single-antenna full-rank models (23) and (24). We now consider the case where $(n-1)$ additional antennas are setup in the vicinity of the single-antenna $r$, thus forming an $n$-dimensional array of multiple antennas (Figure 3). As shown by previous contributions, such an array-based setup proves to be very beneficial to GNSS precise positioning and integrity monitoring, see e.g. [25], [36]-[38]. We now explore the potential benefits of using the array-based setup for TEC determination. For the whole $n$ array antennas, the array observation matrices are defined as $\boldsymbol{\Phi}=\left[\boldsymbol{\phi}_{1}, \ldots, \phi_{n}\right]$ and $\boldsymbol{P}=\left[\boldsymbol{p}_{1}, \ldots, \boldsymbol{p}_{n}\right]$. The distances between the antennas are assumed to be short enough so that the same ionospheric delays, of each satellite, are experienced by all the antennas. Depending on the applications and the environmental conditions, the maximum allowed distance between the antennas may vary. The multivariate formulation of the observation equations (20) reads then

$$
\begin{aligned}
& \boldsymbol{\Phi}(t)=-\left(\boldsymbol{\mu} \otimes \boldsymbol{I}_{m}\right) \boldsymbol{i}_{r}(t) \boldsymbol{e}_{n}^{T}+\left(\boldsymbol{e} \otimes \boldsymbol{I}_{m}\right) \boldsymbol{\rho}(t)+\mathcal{A} \\
& \boldsymbol{P}(t)=+\left(\boldsymbol{\mu} \otimes \boldsymbol{I}_{m}\right) \boldsymbol{i}_{r}(t) \boldsymbol{e}_{n}^{T}+\left(\boldsymbol{e} \otimes \boldsymbol{I}_{m}\right) \boldsymbol{\rho}(t)+\mathcal{D}
\end{aligned}
$$

where

$$
\begin{aligned}
\boldsymbol{\rho} & =\left[\boldsymbol{\rho}_{1}, \ldots, \boldsymbol{\rho}_{n}\right] ; \quad \mathcal{D}=\left[\boldsymbol{d}_{1}, \ldots, \boldsymbol{d}_{n}\right] \\
\mathcal{A} & =\left[\boldsymbol{a}_{1}, \ldots, \boldsymbol{a}_{n}\right]
\end{aligned}
$$

The multivariate observation equations (46) can be linked to the single-antenna full-rank models (23) and (24) through an application of a proper one-to-one transformation to the observation matrices $\boldsymbol{\Phi}$ and $\boldsymbol{P}$. To see this, consider the invertible $n \times n$ matrix

$$
\left[\boldsymbol{u}_{r}, \boldsymbol{D}_{n}\right], \quad \text { with }\left[\boldsymbol{u}_{r}, \boldsymbol{D}_{n}\right]^{-1}=\left[\boldsymbol{e}_{n}, \boldsymbol{C}_{n}\right]^{T}
$$

in which the $n \times(n-1)$ matrix $\boldsymbol{D}_{n}$ denotes the between-antenna differencing matrix [39]. The $n$-vector $\boldsymbol{u}_{r}$ contains zeros, except its $r^{t h}$ element equal to 1 . The $n \times(n-1)$ matrix $\boldsymbol{C}_{n}$ is structured by eliminating the $r^{\text {th }}$ column of the identity matrix $\boldsymbol{I}_{n}$. Thus both $\boldsymbol{u}_{r}$ and $\boldsymbol{C}_{n}$ form the columns of $\boldsymbol{I}_{n}$. Application of (48) to the observation matrices gives

$$
\begin{aligned}
& \boldsymbol{\Phi}(t)\left[\boldsymbol{u}_{r}, \boldsymbol{D}_{n}\right]=\left[\boldsymbol{\phi}_{r}(t), \boldsymbol{\Phi}(t) \boldsymbol{D}_{n}\right] \\
& \boldsymbol{P}(t)\left[\boldsymbol{u}_{r}, \boldsymbol{D}_{n}\right]=\left[\boldsymbol{p}_{r}(t), \quad \boldsymbol{P}(t) \boldsymbol{D}_{n}\right]
\end{aligned}
$$


This shows that the provision of $(n-1)$ extra antennas extends the single-antenna observations $\phi_{r}$ and $\boldsymbol{p}_{r}$ by the betweenantenna differenced observations $\boldsymbol{\Phi} \boldsymbol{D}_{n}$ and $\boldsymbol{P} \boldsymbol{D}_{n}$. The single-antenna full-rank models (23) and (24) are therefore aided by the extra observation equations

$$
\begin{aligned}
& \boldsymbol{\Phi}(t) \boldsymbol{D}_{n}=\left(\boldsymbol{e} \otimes \boldsymbol{I}_{m}\right) \boldsymbol{\rho}(t) \boldsymbol{D}_{n}+\mathcal{A} \boldsymbol{D}_{n} \\
& \boldsymbol{P}(t) \boldsymbol{D}_{n}=\left(\boldsymbol{e} \otimes \boldsymbol{I}_{m}\right) \boldsymbol{\rho}(t) \boldsymbol{D}_{n}+\mathcal{D} \boldsymbol{D}_{n}
\end{aligned}
$$

The above equations follow through post-multiplying (46) by $\boldsymbol{D}_{n}$, along with $\boldsymbol{e}_{n}^{T} \boldsymbol{D}_{n}=0$.

As shown, the extra observation equations (50) do not contain the ionospheric parameters $\boldsymbol{i}_{r}$. One may then wonder how these equations can aid the single-antenna observation equations (23) and (24). The answer lies in their non-zero correlation with (23) and (24). It is this non-zero correlation that improves the precision of the single-antenna solutions $\hat{\boldsymbol{i}}_{r ; \phi}$ and $\hat{\boldsymbol{i}}_{r ; p}$. The unknown parameters, involved in (50), can be further eliminated by forming linear combinations of $\boldsymbol{\Phi} \boldsymbol{D}_{n}$ and $\boldsymbol{P} \boldsymbol{D}_{n}$. Such linear combinations increase the single-antenna model's redundancy by imposing redundant conditions on the ionospheric solutions. For instance, consider the GF combination $\boldsymbol{\mu}_{G F}^{T} \boldsymbol{p}_{q}^{s}(1 t)$ of the aiding antenna $q, q \neq r$ (cf. 19). Since $i_{q}^{s}=i_{r}^{s}$, this combination has the same mean as that of its counterpart of the antenna $r$, that is

$$
\begin{aligned}
\boldsymbol{\mu}_{G F}^{T}\left\{\boldsymbol{p}_{q}^{s}(1 t)-\boldsymbol{p}_{r}^{s}(1 t)\right\} & =\boldsymbol{\mu}_{G F}^{T} \boldsymbol{p}_{q}^{s}(1 t)-\boldsymbol{\mu}_{G F}^{T} \boldsymbol{p}_{r}^{s}(1 t) \\
& =i_{q}^{s}(1 t)-i_{r}^{s}(1 t)=0
\end{aligned}
$$

These linear combinations are examples of the condition equations [40]. Imposing these equations on the solutions (28) and (30) does further adjust them, thereby improving their precision. The multivariate ionospheric condition equations are presented below.

Lemma 2: (Array-aided ionospheric condition equations) The $k$-epoch single-antenna ionospheric solutions (28) and (30) are aided by the following array's condition equations

$$
\begin{aligned}
& \left(\boldsymbol{D}_{f}^{T} \otimes \boldsymbol{D}_{m}^{T}\right) \boldsymbol{\Phi}(\bar{t}) \boldsymbol{D}_{n}=\left(\boldsymbol{D}_{f}^{T} \boldsymbol{\Lambda} \otimes \boldsymbol{I}_{m-1}\right) \boldsymbol{Z} \\
& \left(\boldsymbol{D}_{f}^{T} \otimes \boldsymbol{D}_{m}^{T}\right) \boldsymbol{P}(\bar{t}) \boldsymbol{D}_{n}=0
\end{aligned}
$$

and

$$
\begin{array}{lll}
(\boldsymbol{P}(1 t)-\boldsymbol{\Phi}(1 t)) \boldsymbol{D}_{n} & = & 0 \\
\left(\boldsymbol{D}_{f}^{T} \otimes \boldsymbol{I}_{m}\right)(\epsilon \boldsymbol{P}(1 t)+\boldsymbol{\Phi}(1 t)) \boldsymbol{D}_{n} & = & 0
\end{array}
$$

for $t=2, \ldots, k$.

The integer-valued ambiguity matrix $Z$ is defined as

$$
\boldsymbol{Z}:=\left(\boldsymbol{\Lambda}^{-1} \otimes \boldsymbol{D}_{m}^{T}\right) \mathcal{A} \boldsymbol{D}_{n}
$$

with the $f \times f$ diagonal matrix $\Lambda$ containing the wavelengths that transform the ambiguities $\mathcal{A}$ from units of range to cycles. $\boldsymbol{D}_{f}$ and $\boldsymbol{D}_{m}$ are the between-frequency and between-satellite differencing matrices, respectively.

Proof: see Appendix.

So as to show the contribution of the array to the ionospheric solutions, the array-aided ionospheric condition equations are partitioned into the time-averaged and -differenced components. Note also the presence of the unknown, but integervalued, double-differenced ambiguities $Z$ in the first expression of (52). As long as these ambiguities remain unresolved, the corresponding condition equations do not contribute to the ionospheric solutions. One may therefore aim to resolve $Z$ by performing 'ambiguity-resolution' [24], [25]. We hereafter distinguish the following two different solutions:

- Fixed-solution: It refers to those that are obtained by assuming the integer-valued parameters $Z$ are resolved and known.

- Float-solution: It refers to those that are obtained by assuming the integer-valued parameters $Z$ remain unknown.

In the following, the fixed solutions are distinguished by the --symbol from their float counterparts. The float solutions are

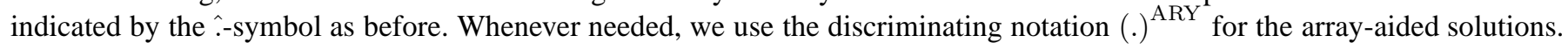
If there is no reason for confusion, we will use the same notation for both the single-antenna and array-aided solutions. The role played by the array-aided condition equations, in improving the ionospheric solutions, is quantified below.

Theorem 2: (Ionospheric observables: array-aided) Extending the full-rank models (23) and (24) by the condition equations (52) and (53), the array-aided time-averaged solution of $\boldsymbol{i}_{r ; \diamond}(\bar{t})$ and its variance matrix are given as

$$
\begin{array}{lll}
\text { Float: } & \hat{\boldsymbol{i}}_{r ; \diamond}^{\mathrm{ARY}}(\bar{t})= \begin{cases}\hat{\boldsymbol{i}}_{r ; \phi}(\bar{t}) ; & \diamond=\phi \\
\mathcal{P}_{e_{m}} \hat{\boldsymbol{i}}_{r ; p}(\bar{t})+\mathcal{P}_{e_{m}}^{\perp} \hat{\boldsymbol{i}}_{\bar{r} ; p}(\bar{t}) ; & \diamond=p\end{cases} \\
\text { Fixed: } & \check{\boldsymbol{i}}_{r ; \diamond}^{\mathrm{ARY}}(\bar{t})= \begin{cases}\mathcal{P}_{e_{m}} \hat{\boldsymbol{i}}_{r ; \phi}(\bar{t})+\mathcal{P}_{e_{m}}^{\perp} \hat{\boldsymbol{i}}_{\bar{r} ; \phi}(\bar{t}) ; & \diamond=\phi \\
\mathcal{P}_{e_{m}} \hat{\boldsymbol{i}}_{r ; p}(\bar{t})+\mathcal{P}_{e_{m}}^{\perp} \hat{\boldsymbol{i}}_{\bar{r} ; p}(\bar{t}) ; & \diamond=p\end{cases}
\end{array}
$$




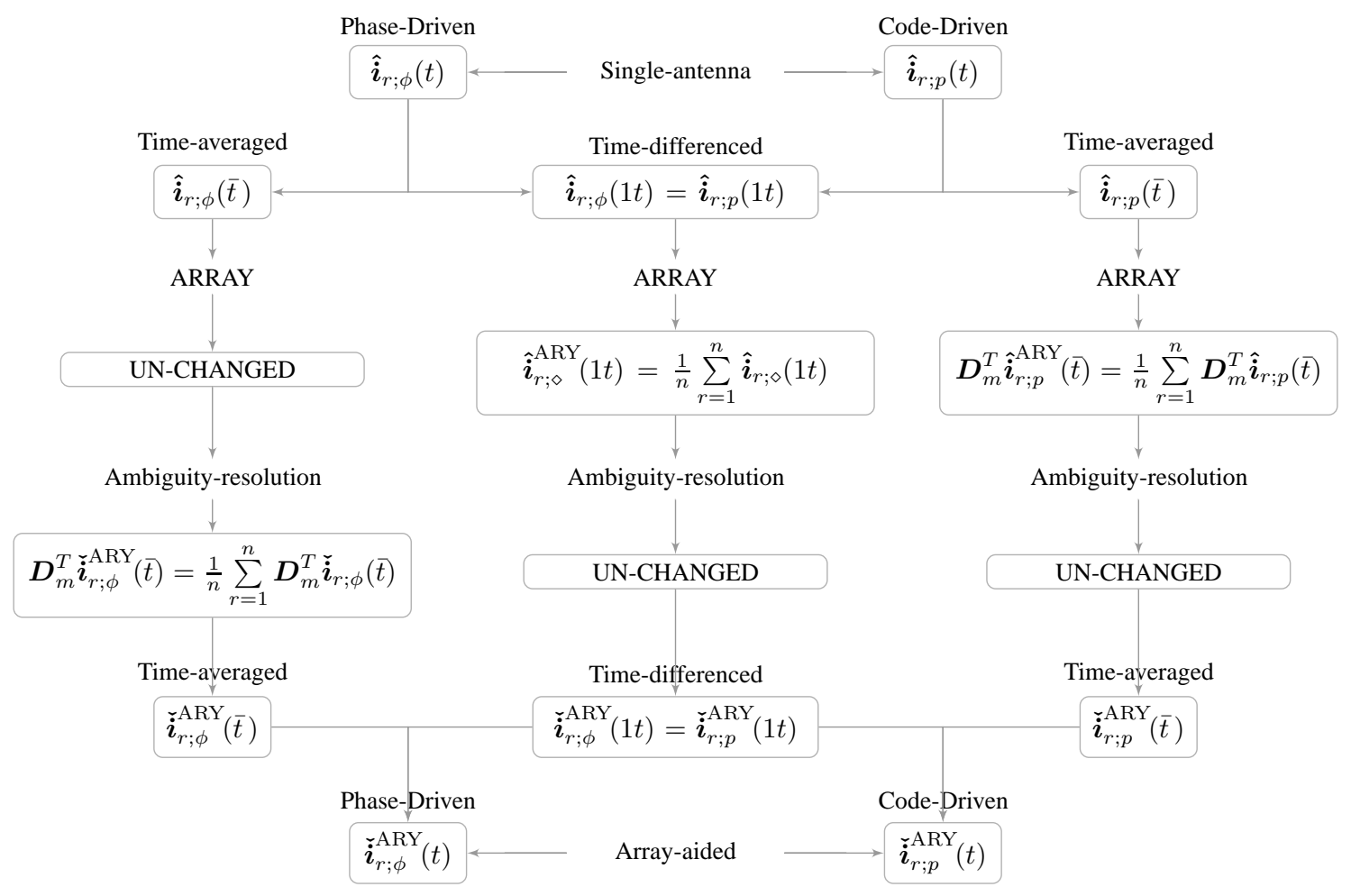

Fig. 4. Array's contribution to the single-antenna ionospheric observables $\hat{\boldsymbol{i}}_{r ; \phi}(t)$ and $\hat{\boldsymbol{i}}_{r ; p}(t)$. The role of array ambiguity resolution in improving the solutions of the time-averaged and -differenced components is also illustrated.

and

$$
\begin{aligned}
& \text { Float: } \quad \mathrm{D}\left(\hat{\boldsymbol{i}}_{r ; \diamond}^{\mathrm{ARY}}(\bar{t})\right)= \begin{cases}\mathrm{D}\left(\hat{\boldsymbol{i}}_{r ; \phi}(\bar{t})\right) ; \\
\mathcal{P}_{e_{m}} \mathrm{D}\left(\hat{\boldsymbol{i}}_{r ; p}(\bar{t})\right)+\frac{1}{n} \mathcal{P}_{e_{m}}^{\perp} \mathrm{D}\left(\hat{\boldsymbol{i}}_{r ; p}(\bar{t})\right) ; & \diamond=p\end{cases} \\
& \text { Fixed: } \quad \mathrm{D}\left(\check{\boldsymbol{i}}_{r ; \diamond}^{\mathrm{ARY}}(\bar{t})\right)= \begin{cases}\mathcal{P}_{e_{m}} \mathrm{D}\left(\hat{\boldsymbol{i}}_{r ; \phi}(\bar{t})\right)+\frac{1}{n} \mathcal{P}_{e_{m}}^{\perp} \mathrm{D}\left(\hat{\boldsymbol{i}}_{r ; \phi}(\bar{t})\right) ; & \diamond=\phi \\
\mathcal{P}_{e_{m}} \mathrm{D}\left(\hat{\boldsymbol{i}}_{r ; p}(\bar{t})\right)+\frac{1}{n} \mathcal{P}_{e_{m}}^{\perp} \mathrm{D}\left(\hat{\boldsymbol{i}}_{r ; p}(\bar{t})\right) ; & \diamond=p\end{cases}
\end{aligned}
$$

While the array-aided time-differenced solutions of $\boldsymbol{i}_{r ; \diamond}(1 t)$ and their (co)variance matrices are given as

$$
\check{\boldsymbol{i}}_{r ; \diamond}^{\mathrm{ARY}}(1 t)=\hat{\boldsymbol{i}}_{r ; \diamond}^{\mathrm{ARY}}(1 t)=\hat{\boldsymbol{i}}_{\bar{r} ; \diamond}(1 t) ; \diamond=\{\phi, p\}
$$

and

$$
\mathrm{C}\left(\hat{\boldsymbol{i}}_{r ; \diamond}^{\mathrm{ARY}}(1 t), \hat{\boldsymbol{i}}_{r ; \diamond}^{\mathrm{ARY}}(1 l)\right)=\frac{1}{n} \mathrm{C}\left(\hat{\boldsymbol{i}}_{r ; \diamond}(1 t), \hat{\boldsymbol{i}}_{r ; \diamond}(1 l)\right)
$$

The antenna-averaging operator is defined as $(.)_{\bar{r}}=\frac{1}{n} \sum_{r=1}^{n}(.)_{r}$, while the projection matrices $\mathcal{P}_{e_{m}}$ and $\mathcal{P}_{e_{m}}^{\perp}$ are given by

Proof: see Appendix.

$$
\mathcal{P}_{e_{m}}=\frac{1}{\boldsymbol{e}_{m}^{T} \overline{\boldsymbol{W}} \boldsymbol{e}_{m}} \boldsymbol{e}_{m} \boldsymbol{e}_{m}^{T} \overline{\boldsymbol{W}} ; \quad \mathcal{P}_{e_{m}}^{\perp}=\boldsymbol{I}_{m}-\mathcal{P}_{e_{m}}
$$

Theorem 2 conveys five important messages (see Figure 4). First, the array-aided solutions $\check{\boldsymbol{i}}_{r ; p}^{\mathrm{ARY}}(\bar{t})$ and $\check{\boldsymbol{i}}_{r ; \phi}^{\mathrm{ARY}}(\bar{t})$ do not follow as an 'antenna-averaged' version of the single-antenna solutions $\hat{\boldsymbol{i}}_{r ; p}(\bar{t})$ and $\hat{\boldsymbol{i}}_{r ; \phi}(\bar{t})$. Pre-multiplying the second and third expressions of (55) by $\boldsymbol{D}_{m}^{T}$, together with $\boldsymbol{D}_{m}^{T} \mathcal{P}_{e_{m}}=0$ and $\boldsymbol{D}_{m}^{T} \mathcal{P}_{e_{m}}^{\perp}=\boldsymbol{D}_{m}^{T}$, gives, however, a different picture for their between-satellite differenced combinations, that is

$$
\boldsymbol{D}_{m}^{T} \check{\boldsymbol{i}}_{r ; \diamond}^{\mathrm{ARY}}(\bar{t})=\boldsymbol{D}_{m}^{T} \hat{\boldsymbol{i}}_{\bar{r} ; \diamond}(\bar{t}) ; \quad \diamond=\{\phi, p\}
$$

Thus, only for their between-satellite differenced combinations, antenna-averaging applies. This is what one would expect. Due to the presence of the unknown between-antenna receiver biases $\mathcal{A} \boldsymbol{D}_{n}$ and $\mathcal{D} \boldsymbol{D}_{n}$, introduced in (50), the extra antennas' data cannot fully contribute to the ionospheric solutions. This notion is visualised in Figure 5 (a) where the between-antenna receiver DCBs $\mathcal{D} \boldsymbol{D}_{n}$ are depicted as the blue blocks. As these biases are eliminated through between-satellite differencing, the between-satellite differenced combinations of the extra antennas' data would however contribute to the ionospheric solutions. 

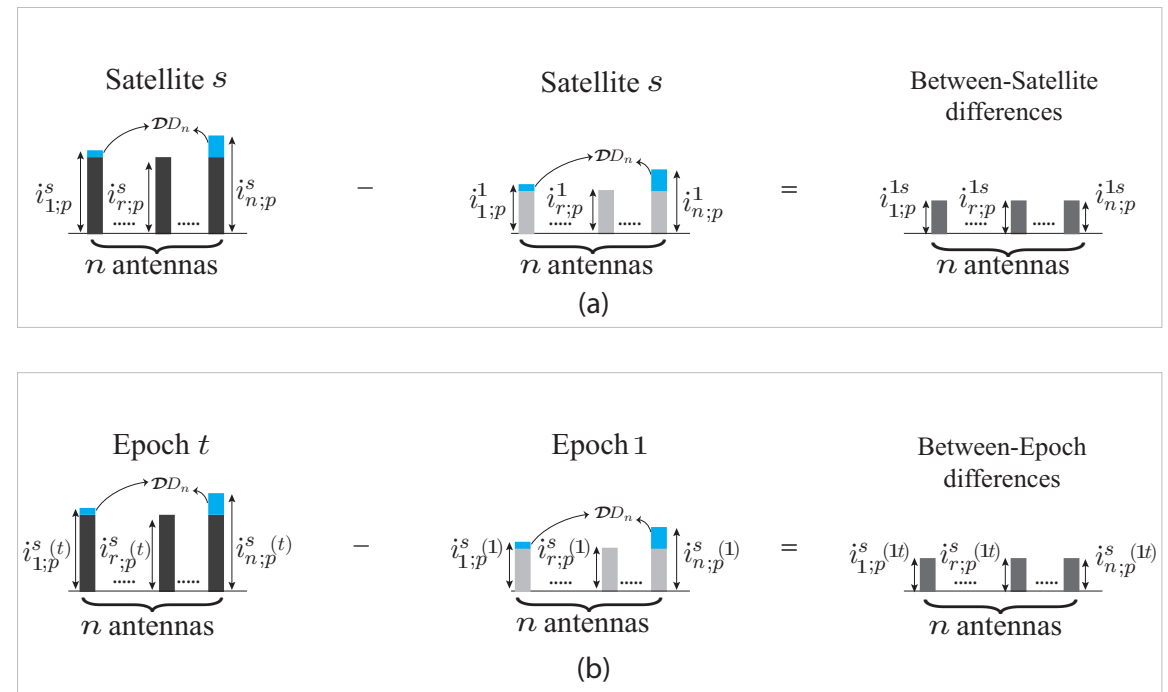

Fig. 5. Visualisation of the between-satellite (a) and between-epoch (b) differences of the array-aided ionospheric solutions. Taking antenna $r$ as reference, the between-antenna receiver DCBs $\mathcal{D} \boldsymbol{D}_{n}$ are depicted as the blue blocks. Thus there is no blue block (i.e. between-antenna receiver DCBs) for antenna $r$ by definition. As these biases are eliminated through differencing, the differenced solutions $i_{l ; p}^{1 s}=i_{l ; p}^{s}-i_{l ; p}^{1}(l=1, \ldots, n)$ would then represent $n$ quantities (of the same mean). Thus their array-aided version follows as their 'antenna-average'. The same conclusion is drawn for the time-differenced solutions $i_{l ; p}^{s}(1 t)=i_{l ; p}^{s}(t)-i_{l ; p}^{s}(1)(l=1, \ldots, n)$.

Second, in contrast to the time-averaged solutions, the time-differenced solutions $\hat{\boldsymbol{i}}_{r ; \phi}^{\mathrm{ARY}}(1 t)$ and $\hat{\boldsymbol{i}}_{r ; p}^{\mathrm{ARY}}(1 t)$ simply follow from the 'antenna-averaged' version of their single-antenna counterparts $\hat{\boldsymbol{i}}_{r ; \phi}(1 t)$ and $\hat{\boldsymbol{i}}_{r ; p}(1 t)$. This is because of the underlying assumption stating that the ambiguity and code biases $\mathcal{A}$ and $\mathcal{D}$ behave constant in time. To better appreciate this, we illustrate the corresponding mechanism in Figure 5 (b). As these biases are eliminated through between-epoch differencing, the timedifferenced combinations of the extra antennas' data fully contribute to the ionospheric solutions. Since the time-differenced components $\hat{\boldsymbol{i}}_{r ; p}(1 t)(r=1, \ldots, n)$ represent $n$ quantities (of the same mean), their array-aided version follows as their 'antenna-average'.

Third, according to (56), the precision of the array-aided solution $\check{\boldsymbol{i}}_{r ; p}^{\mathrm{ARY}}(\bar{t})$ outperforms that of the single-antenna solution $\hat{\boldsymbol{i}}_{r ; p}(\bar{t})$. Since the code-driven time-averaged solutions contain information on the satellite DCBs (cf. Table II), the precision of corresponding array-aided satellite DCB solutions does also outperform its single-antenna counterpart. Similar to the first remark (cf. 60), the array-aided satellite DCBs do not, however, follow as the 'antenna-average' of their single-antenna versions. Thus only for their between-satellite differences, antenna-averaging applies.

Fourth, the time-averaged solutions $\hat{\boldsymbol{i}}_{r ; \phi}(\bar{t})$ and $\hat{\boldsymbol{i}}_{r ; p}(\bar{t})$ are shown to have completely different responses to array ambiguityresolution. While the float code-driven solution $\hat{\boldsymbol{i}}_{r ; p}^{\mathrm{ARY}}(\bar{t})$ remains unaffected after ambiguity-resolution, that of the phase-driven $\hat{\boldsymbol{i}}_{r ; \phi}^{\mathrm{ARY}}(\bar{t})$ improves to its fixed counterpart. The float code-driven solution $\hat{\boldsymbol{i}}_{r ; p}^{\mathrm{ARY}}(\bar{t})$ has already benefited from the data of the extra $(n-1)$ antennas $(1 \ldots, r-1, r+1, \ldots, n)$. Thus, there is no difference between its float and fixed solutions. The phasedriven solution $\hat{\boldsymbol{i}}_{r ; \phi}^{\mathrm{ARY}}(\bar{t})$ does, however, not benefit from the data of the extra antennas, unless array ambiguity-resolution is applied.

Fifth, as (57) shows, the time-differenced solutions $\hat{\boldsymbol{i}}_{r ; \phi}^{\mathrm{ARY}}(1 t)=\hat{\boldsymbol{i}}_{r ; p}^{\mathrm{ARY}}(1 t)$ are insensitive to array ambiguity-resolution. To address this, we recall that the ambiguity condition equations $Z$ (first expression of 52) are functions of the time-averaged data. Being uncorrelated with the time-differenced data, they do therefore not contribute to the time-differenced solutions.

\section{B. Improved precision of the ionospheric observables}

The precision of the array-aided ionospheric solutions $\hat{\boldsymbol{i}}_{r ; \phi}^{\mathrm{ARY}}(t)$ and $\hat{\boldsymbol{i}}_{r ; p}^{\mathrm{ARY}}(t)$ can be evaluated by an application of Theorem 2.

Corollary 4: (Precision of the ionospheric observables: array-aided) Aided by the condition equations (52) and (53), the full rank models (23) and (24), lead to the (co)variance matrices of the array-aided ionospheric observables as $(\diamond=\{\phi, p\})$

Float: $\quad \mathrm{C}\left(\hat{\boldsymbol{i}}_{r ; \diamond}^{\mathrm{ARY}}(t), \hat{\boldsymbol{i}}_{r ; \diamond}^{\mathrm{ARY}}(l)\right)=\mathrm{D}\left(\hat{\boldsymbol{i}}_{r ; \diamond}^{\mathrm{ARY}}(\bar{t})\right)+\frac{\sigma_{\phi}^{2}}{n f \gamma}[1+\epsilon]\left(\delta_{t l} \boldsymbol{W}_{t}^{-1}-\frac{1}{k} \overline{\boldsymbol{W}}^{-1}\right)$

Fixed: $\quad \mathrm{C}\left(\check{\boldsymbol{i}}_{r ; \diamond}^{\mathrm{ARY}}(t), \check{\boldsymbol{i}}_{r ; \diamond}^{\mathrm{ARY}}(l)\right)=\mathrm{D}\left(\check{\boldsymbol{i}}_{r ; \diamond}^{\mathrm{ARY}}(\bar{t})\right)+\frac{\sigma_{\phi}^{2}}{n f \gamma}[1+\epsilon]\left(\delta_{t l} \boldsymbol{W}_{t}^{-1}-\frac{1}{k} \overline{\boldsymbol{W}}^{-1}\right)$ 
TABLE IV

NUMERICAL EVALUATIONS OF THE IONOSPHERIC STANDARD DEVIATION (63), AS A FUNCTION OF THE NUMBER OF ARRAY ANTENNAS $n$ FOR A GPS DUAL-FREQUENCY (L1/L2) DATA-SET, WITH $\sigma_{p} \approx 1.85$ (TECU), $\sigma_{\phi}^{2}=\epsilon \sigma_{p}^{2}(\epsilon=0.0001)$, AND $m=8$. THE RESULTS ARE PRESENTED FOR THE NUMBERS OF EPOCHS $k=1$ AND $k=100$.

\begin{tabular}{|c|c|c|c|c|c|c|c|c|c|c|}
\hline & No. of antennas $(n)$ & 1 & 2 & 3 & 4 & 5 & 6 & 7 & 8 & 9 \\
\hline & $\sigma_{\hat{i}_{r, \phi}^{s}}[$ TECU] $(k=1)$ & 0.04 & 0.04 & 0.04 & 0.04 & 0.04 & 0.04 & 0.04 & 0.04 & 0.04 \\
\hline Float & $\sigma_{\hat{i}_{r ; \phi}^{s}}^{r ; \phi}[$ TECU] $(k=100)$ & 0.04 & 0.03 & 0.02 & 0.02 & 0.02 & 0.02 & 0.02 & 0.01 & 0.01 \\
\hline & $\sigma_{i_{r ; \phi}^{s}}^{s}[$ TECU] $(k=1)$ & 0.04 & 0.03 & 0.03 & 0.02 & 0.02 & 0.02 & 0.02 & 0.02 & 0.02 \\
\hline Fixed & $\sigma_{\check{i}_{r ; \phi}^{s}}^{s}[$ TECU] $(k=100)$ & 0.04 & 0.03 & 0.02 & 0.02 & 0.02 & 0.02 & 0.02 & 0.01 & 0.01 \\
\hline & $\sigma_{\check{i}_{r ; p}^{s}}=\sigma_{\hat{i}_{r ; p}^{s}}[\mathrm{TECU}](k=1)$ & 4.05 & 3.04 & 2.61 & 2.37 & 2.22 & 2.11 & 2.02 & 1.96 & 1.91 \\
\hline Float/Fixed & $\sigma_{\check{i}_{r ; p}^{s}}=\sigma_{\hat{i}_{r ; p}^{s}}[$ TECU] $(k=100)$ & 0.41 & 0.30 & 0.26 & 0.24 & 0.22 & 0.21 & 0.20 & 0.20 & 0.19 \\
\hline
\end{tabular}

where

$$
\begin{gathered}
\mathrm{D}\left(\hat{\boldsymbol{i}}_{r ; \diamond}^{\mathrm{ARY}}(\bar{t})\right)= \begin{cases}\left(\frac{2 \sigma_{\phi}^{2}}{k \mu_{12}^{2}}\right) \overline{\boldsymbol{W}}^{-1} ; & \diamond=\phi \\
\left(\frac{2 \sigma_{p}^{2}}{k \mu_{12}^{2}}\right)\left\{\left[\frac{n-1}{n}\right] \frac{1}{m^{*}} \boldsymbol{e}_{m} \boldsymbol{e}_{m}^{T}+\frac{1}{n} \overline{\boldsymbol{W}}^{-1}\right\} ; & \diamond=p\end{cases} \\
\mathrm{D}\left(\check{\boldsymbol{i}}_{r ; \diamond}^{\mathrm{ARY}}(\bar{t})\right)= \begin{cases}\left(\frac{2 \sigma_{\phi}^{2}}{k \mu_{12}^{2}}\right)\left\{\left[\frac{n-1}{n}\right] \frac{1}{m^{*}} \boldsymbol{e}_{m} \boldsymbol{e}_{m}^{T}+\frac{1}{n} \overline{\boldsymbol{W}}^{-1}\right\} ; & \diamond=\phi \\
\left(\frac{2 \sigma_{p}^{2}}{k \mu_{12}^{2}}\right)\left\{\left[\frac{n-1}{n}\right] \frac{1}{m^{*}} \boldsymbol{e}_{m} \boldsymbol{e}_{m}^{T}+\frac{1}{n} \overline{\boldsymbol{W}}^{-1}\right\} ; & \diamond=p\end{cases}
\end{gathered}
$$

with $m^{*}=\boldsymbol{e}_{m}^{T} \overline{\boldsymbol{W}} \boldsymbol{e}_{m}$.

Proof: Follows by an application of the variance propagation law to (25), together with the equalities (56) and (58). To gain some numerical insight into the precision improvement, brought by employing an array of antennas, the following example is presented.

Example 1: We recall that the square-root of a diagonal element of the variance matrix $\mathrm{D}\left(\hat{\boldsymbol{i}}_{r ; \diamond}(t)\right)$ represents the standard deviation of an individual solution $\hat{i}_{r ; \infty}^{s}(t)$. Let us now assume that no elevation-weighting is applied to a dual-frequency GPS (L1/L2) data-set (i.e. $\left.\boldsymbol{W}_{t}=\boldsymbol{I}_{m}\right)$. Through (61) and the equality $\mathrm{D}\left(\hat{\boldsymbol{i}}_{r ; \diamond}(t)\right)=\mathrm{C}\left(\hat{\boldsymbol{i}}_{r ; \diamond}(t), \hat{\boldsymbol{i}}_{r ; \diamond}(t)\right)$, the stated standard deviation, over $k$ epochs, can be given by

$$
\begin{aligned}
\sigma_{\hat{i}_{r ; \phi}^{s}} & =\sigma_{\phi}\left\{\frac{2}{k \mu_{12}^{2}}+\left[\frac{k-1}{k}\right] \frac{1}{n f \gamma}(1+\epsilon)\right\}^{\frac{1}{2}} \\
\sigma_{\check{i}_{r ; \phi}^{s}} & =\sigma_{\phi}\left\{\frac{2}{k \mu_{12}^{2}}\left[1-\left(\frac{m-1}{m}\right)\left(\frac{n-1}{n}\right)\right]+\left[\frac{k-1}{k}\right] \frac{1}{n f \gamma}(1+\epsilon)\right\}^{\frac{1}{2}} \\
\sigma_{\check{i}_{r ; p}^{s}}=\sigma_{\hat{i}_{r ; p}^{s}} & =\sigma_{p}\left\{\frac{2}{k \mu_{12}^{2}}\left[1-\left(\frac{m-1}{m}\right)\left(\frac{n-1}{n}\right)\right]+\left[\frac{k-1}{k}\right] \frac{\epsilon}{n f \gamma}(1+\epsilon)\right\}^{\frac{1}{2}}
\end{aligned}
$$

We set $\sigma_{p}=30 \mathrm{~cm} \approx 1.85$ TEC unit (TECU) with $\sigma_{\phi}^{2}=\epsilon \sigma_{p}^{2}(\epsilon=0.0001)$, as each TECU roughly causes $16.2 \mathrm{~cm}$ slant delay on L1 of GPS [8]. In case of GPS L1 and L2 frequencies, the coefficients $\mu_{1}=1$ and $\mu_{2}=1.6469$ yield $\mu_{12} \approx 0.6469$.

The number of visible satellites is set to $m=8$. Given these settings, the numerical evaluations of (63), as a function of the number of array antennas, are presented in Table IV. Note that these values represent the 'precision' of the observables and not their 'accuracy'. Their accuracy is further affected by the potential presence of the mis-modeled effects such as e.g., multipath.

As shown, the standard deviations of the phase-driven solutions are smaller than their code-driven counterparts (two orders of magnitude). This corresponds to the square-root of the phase-to-code variance ratio $\epsilon$, i.e. $\sqrt{\epsilon}=0.01$. In the single-epoch case $k=1$, increasing the number of aiding antennas $n$ does not improve the precision of the float phase-driven solutions. For the multi-epoch case $k=100$ however, the stated precision improves as the number of antennas increases. Applying array ambiguity-resolution, an increase in $n$ results in the precision improvement of the fixed phase-driven solutions for both the single-epoch case $k=1$ and the multi-epoch case $k=100$. In case of the code-driven solutions, increasing the number of antennas to $n=9$ gives rise to a $50 \%$ reduction in the size of the corresponding standard deviation.

\section{ARRAY'S RELEVANCE FOR TEC DETERMINATION}

In the previous section, the precision of the ionospheric observables was shown to be improved by extending the data of a single antenna to those of an array of nearby antennas (Figure 3). These ionospheric observables are the least-squares solutions of the estimable slant ionospheric delays, formed by choosing a set of GNSS parameters as the $\mathcal{S}$-basis (cf. Sect. II). We also showed that choosing different $\mathcal{S}$-bases leads to different ionospheric observables of different precision. For instance, the precision of the phase-driven observables $\hat{\boldsymbol{i}}_{r ; \phi}$ is governed by the very precise phase data, whereas that of the code-driven observables $\hat{\boldsymbol{i}}_{r ; p}$ is dominated by the rather poor precision of the code data.

In the context of TEC determination, such precision dependency on the choice of $\mathcal{S}$-basis may lead one to be inclined to prefer the phase-driven ionospheric observables over their code-driven counterparts, see e.g. [41], [42]. It should, however, be 
remarked that what matters is in fact the way these observables are mapped to the unknown TEC sought. This becomes clear, would one adopt an external ionospheric model to capture both the spatial and temporal characteristics of the ionosphere.

\section{A. Single-layer model}

Next to the other ionospheric models as used in the literature [31], a popular ionospheric model for TEC determination is the single-layer model which is also known as the thin-shell model, see e.g. [2], [4], [5], [9], [43]-[45]. Let the ionosphere be represented by a thin shell at a fixed height above the Earth. The vertical TEC $\nu_{r}^{s}$, experienced in the radial direction of the shell, can then be considered as a mapped version of the unbiased slant TEC $i_{r}^{s}$,

$$
i_{r}^{s}(t)=\mathcal{M}_{r}^{s}(t) \nu_{r}^{s}(t), \quad \nu_{r}^{s}(t)=\sum_{u=1}^{q} b_{r, u}^{s}(t) c_{l, u}
$$

for the time intervals

$$
t=(l-1) \tau+1, \ldots, l \tau ; \quad l=1, \ldots, K
$$

with $\mathcal{M}_{r}^{s}$ being an ionospheric mapping function and $\tau$ being the refreshing interval. The known coefficients $b_{r, u}^{s}(u=1, \ldots, q)$ form the ionospheric basis functions. In case of world-wide TEC determination, these basis functions often take the form of spherical harmonic functions [5]. For regional-scale TEC determination, other forms of the basis functions, such as the polynomial functions are also taken [45]. The unknown coefficients $c_{l, u}(u=1, \ldots, q)$ are assumed to be piece-wise constant in time. According to (65), the observational time-span $t=1, \ldots, k$ is partitioned into $K$ time intervals, thus $k=K \tau$. The coefficients $c_{l, u}$ are therefore constant within the $l^{\text {th }}$ time interval of (65).

\section{B. Solution of the unbiased TEC}

To quantify how the ionospheric observables $\hat{\boldsymbol{i}}_{r ; \phi}$ and $\hat{\boldsymbol{i}}_{r ; p}$ contribute to the TEC solution, we again make use of the vectorial presentation. The vectorial version of (64) can be expressed by

$$
\boldsymbol{i}_{r}(t)=\boldsymbol{B}_{l}(t) \boldsymbol{c}_{l} ; \quad t=(l-1) \tau+1, \ldots, l \tau
$$

where

$$
\begin{aligned}
\boldsymbol{B}_{l} & =\left[\boldsymbol{b}_{1}, \ldots, \boldsymbol{b}_{q}\right] ; \quad \boldsymbol{c}_{l}=\left[c_{l, 1}, \ldots, c_{l, q}\right]^{T} \\
\boldsymbol{b}_{u} & =\left[\mathcal{M}_{r}^{1} b_{r, u}^{1}, \ldots, \mathcal{M}_{r}^{m} b_{r, u}^{m}\right]^{T}
\end{aligned}
$$

Using the interpretations given in (9) and (15), the ionospheric observables $\hat{\boldsymbol{i}}_{r ; \phi}$ and $\hat{\boldsymbol{i}}_{r ; p}$ are linked to the model (66) as follows

$$
\hat{\boldsymbol{i}}_{r ; \diamond}(t)=\boldsymbol{B}_{l}(t) \boldsymbol{c}_{l}+\left(\boldsymbol{e}_{\tau} \otimes \boldsymbol{I}_{m}\right) \boldsymbol{\nabla}_{; \diamond} ; \diamond=\{\phi, p\}
$$

with

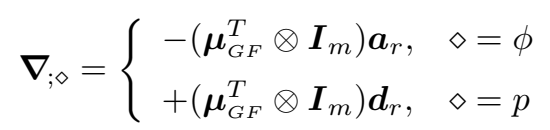

Thus when the phase-driven solution is taken, the unknown bias vector $\nabla_{; \phi}$ represents the GF combinations of ambiguities $\boldsymbol{a}_{r}$. In case of the code-driven solution, $\nabla_{; p}$ would represent those of the code biases $\boldsymbol{d}_{r}$.

Definition 2: (Single-layer full-rank models) Collecting equations (68) of all the $K$ time intervals, the phase-driven and code-driven full-rank models of the single-layer model (64) are defined as $(\diamond=\{\phi, p\})$

$$
\left[\begin{array}{c}
\hat{\boldsymbol{i}}_{r ; \diamond[1]} \\
\vdots \\
\hat{\boldsymbol{i}}_{r ; \diamond[K]}
\end{array}\right]=\left[\begin{array}{c}
\boldsymbol{B}_{[1]} \\
\vdots \\
\boldsymbol{B}_{[K]}
\end{array}\right] \boldsymbol{c}+\left[\begin{array}{c}
\boldsymbol{A} \\
\vdots \\
\boldsymbol{A}
\end{array}\right] \boldsymbol{\nabla}_{; \diamond}
$$

where $\boldsymbol{A}=\left(\boldsymbol{e}_{\tau} \otimes \boldsymbol{I}_{m}\right), \boldsymbol{c}=\left[\boldsymbol{c}_{1}^{T}, \ldots, \boldsymbol{c}_{K}^{T}\right]^{T}$ and

$$
\begin{aligned}
& \boldsymbol{B}_{[1]}=\left[\boldsymbol{B}_{1}, 0, \ldots, 0,0, \ldots, 0\right] \\
& \boldsymbol{B}_{[l]}=\left[0, \ldots, 0, \boldsymbol{B}_{l}, 0, \ldots, 0\right] \\
& \boldsymbol{B}_{[K]}=\left[0, \ldots, 0,0, \ldots, 0, \boldsymbol{B}_{K}\right]
\end{aligned}
$$

for $l=1, \ldots, K$. The observation vector $\hat{\boldsymbol{i}}_{r ; \diamond[l]}$ contains all the solutions $\hat{\boldsymbol{i}}_{r ; \diamond}(t)$ within the $l^{\text {th }}$ time interval (65). With the full-rank model (70), we are now in a position to present the least-squares solution for $c$, thus for its linear functions, namely, the unbiased slant TEC $\boldsymbol{i}_{r}$. 


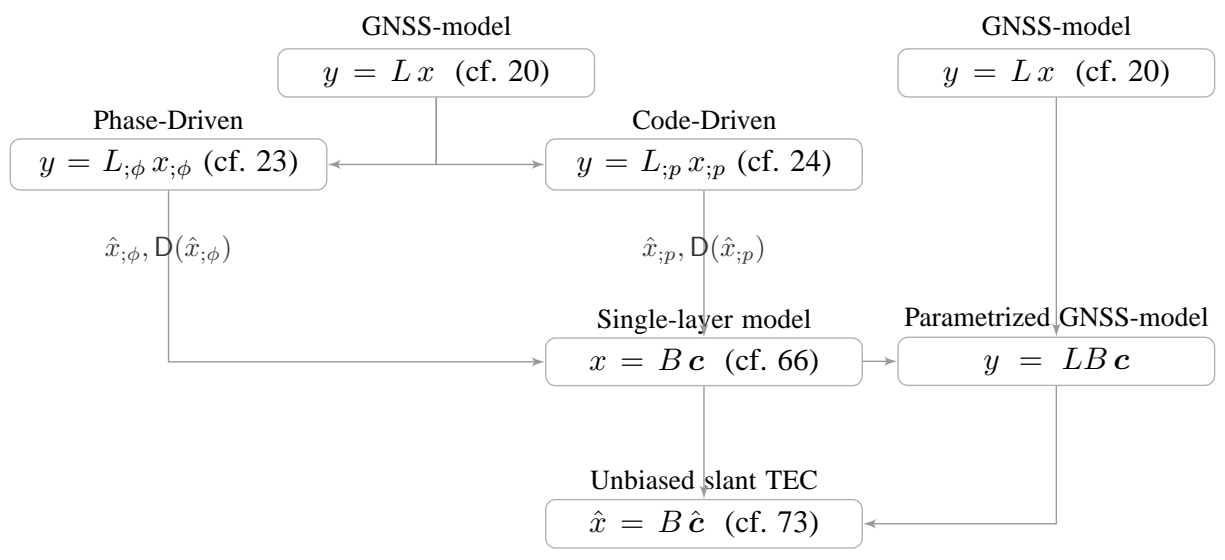

Fig. 6. Diagram showing the invariance property of the TEC solutions irrespective of the types of the ionospheric observables, provided that the inverse of the variance matrices of the observables is taken as the weight matrix of the underlying least-squares adjustment. Such TEC solutions are identical to those obtained by parametrizing the GNSS model (20) using the single-layer model (66) and determining the TEC solutions in one go.

Theorem 3: (Invariance property of the TEC solutions) Irrespective of whether the phase-driven ionospheric observables $\hat{\boldsymbol{i}}_{r ; \phi}$ or their code-driven counterparts $\hat{\boldsymbol{i}}_{r ; p}$ are taken as the input, the full-rank model (70) results in a unique set of least-squares normal equations

$$
\boldsymbol{N} \hat{\boldsymbol{c}}=\boldsymbol{y}, \quad \text { with } \quad \mathrm{D}(\hat{\boldsymbol{c}})=\frac{\sigma_{\phi}^{2}}{n f \gamma}(1+\epsilon) \boldsymbol{N}^{-1}
$$

with $\gamma=(1+\epsilon)^{2} \sigma_{\mu}^{2}+4 \epsilon \bar{\mu}^{2}$.

The normal matrix $\boldsymbol{N}$ and its right-hand side vector $\boldsymbol{y}$ are given in (102) (Appendix). The right-hand side vector $\boldsymbol{y}$ is a function of the time-differenced components $\hat{\boldsymbol{i}}_{r ; \phi}(1 t)=\hat{\boldsymbol{i}}_{r ; p}(1 t)$ only, thereby invariant for the choice of observables $\hat{\boldsymbol{i}}_{r ; \diamond}(\diamond=\{\phi, p\})$.

Proof: see Appendix.

According to Theorem 3, it is the time-differenced components $\hat{\boldsymbol{i}}_{r ; \phi}(1 t)=\hat{\boldsymbol{i}}_{r ; p}(1 t)$ that govern the least-squares solutions $\hat{\boldsymbol{c}}_{l}$ and any linear functions thereof, including the slant TEC solutions (cf. 66)

$$
\hat{\boldsymbol{i}}_{r}(t)=\boldsymbol{B}_{l}(t) \hat{\boldsymbol{c}}_{l} ; \quad t=(l-1) \tau+1, \ldots, l \tau
$$

Thus, the difference in the precision of the phase- and code-driven ionospheric observables (cf. 61) is not relevant to TEC determination. No matter which set of $\mathcal{S}$-basis is chosen, the TEC solution $\hat{\boldsymbol{i}}_{r}(t)$ and its precision remain invariant, provided that a rigorous least-squares adjustment is applied. The term 'rigorous' refers to case where the inverse of the variance matrices of the observables, i.e. $\mathrm{D}\left(\hat{\boldsymbol{i}}_{; \phi}\right)$ and $\mathrm{D}\left(\hat{\boldsymbol{i}}_{; p}\right)$, is taken as the weight matrix of the underlying least-squares adjustment. Such TEC solutions are identical to those obtained by parametrizing the GNSS model (20) using the single-layer model (66) (Figure 6). With such a parameterized model, the underlying ionospheric rank-deficiency would vanish, thereby allowing one to determine the unbiased TEC parameters in one go.

As the second expression of (72) shows, the precision of $\hat{\boldsymbol{c}}_{l}$ and therefore that of the TEC solution $\hat{\boldsymbol{i}}_{r}(t)$ are governed by the precision of the phase data. Both the number of frequencies $f$ and the number of antennas $n$ also play a prominent role in improving the precision of the TEC. To show the role of the number of frequencies $f$ on the TEC precision, let us first make some approximation. Neglecting the small value $\epsilon$, one gets in (72) the approximation $\gamma \approx \sigma_{\mu}^{2}$. Therefore the TEC variance approximately behaves as (for a fixed number of epochs)

$$
\mathrm{D}\left(\hat{\boldsymbol{i}}_{r}(t)\right) \dot{\propto} \frac{\sigma_{\phi}^{2}}{n f \sigma_{\mu}^{2}}
$$

where the notation $\dot{\propto}$ means 'almost proportional to'. Thus next to the carrier-phase variance $\sigma_{\phi}^{2}$, it also depends on the number of antennas $n$, number of frequencies $f$ and the dispersion of the frequency bands $\sigma_{\mu}^{2}$. The larger the frequency band spacing, the larger the quantity $\sigma_{\mu}^{2}$, thus the smaller the TEC variance becomes. As the TEC variance is reversely proportional to $n$ and $f$, the TEC variance decreases as the number of antennas/frequencies increases. We remark that (74) holds for the 'first-order' term of the slant ionospheric delays as introduced in (1). The other ionospheric higher-order effects [46] are not considered here. The following example provides further numerical insight.

Example 2: (Bi-quadratic basis functions) Let the vertical TEC $\nu_{r}^{s}$, in (64), be represented by a bi-quadratic expansion [9]

$$
\nu_{r}^{s}=c_{l, 1}+x_{r}^{s} c_{l, 2}+y_{r}^{s} c_{l, 3}+\left(x_{r}^{s}\right)^{2} c_{l, 4}+\left(y_{r}^{s}\right)^{2} c_{l, 5}+x_{r}^{s} y_{r}^{s} c_{l, 6}
$$

Thus, the role of the basis functions $b_{r, u}^{s}$ is taken by $1, x_{r}^{s}, y_{r}^{s},\left(x_{r}^{s}\right)^{2},\left(y_{r}^{s}\right)^{2}$ and $x_{r}^{s} y_{r}^{s}$, i.e. $q=6$. They are formed as

$$
x_{r}^{s}=\left(\alpha^{s}-\alpha_{r}\right) \cos \left(\beta_{r}\right), \quad y_{r}^{s}=\left(\beta^{s}-\beta_{r}\right)
$$



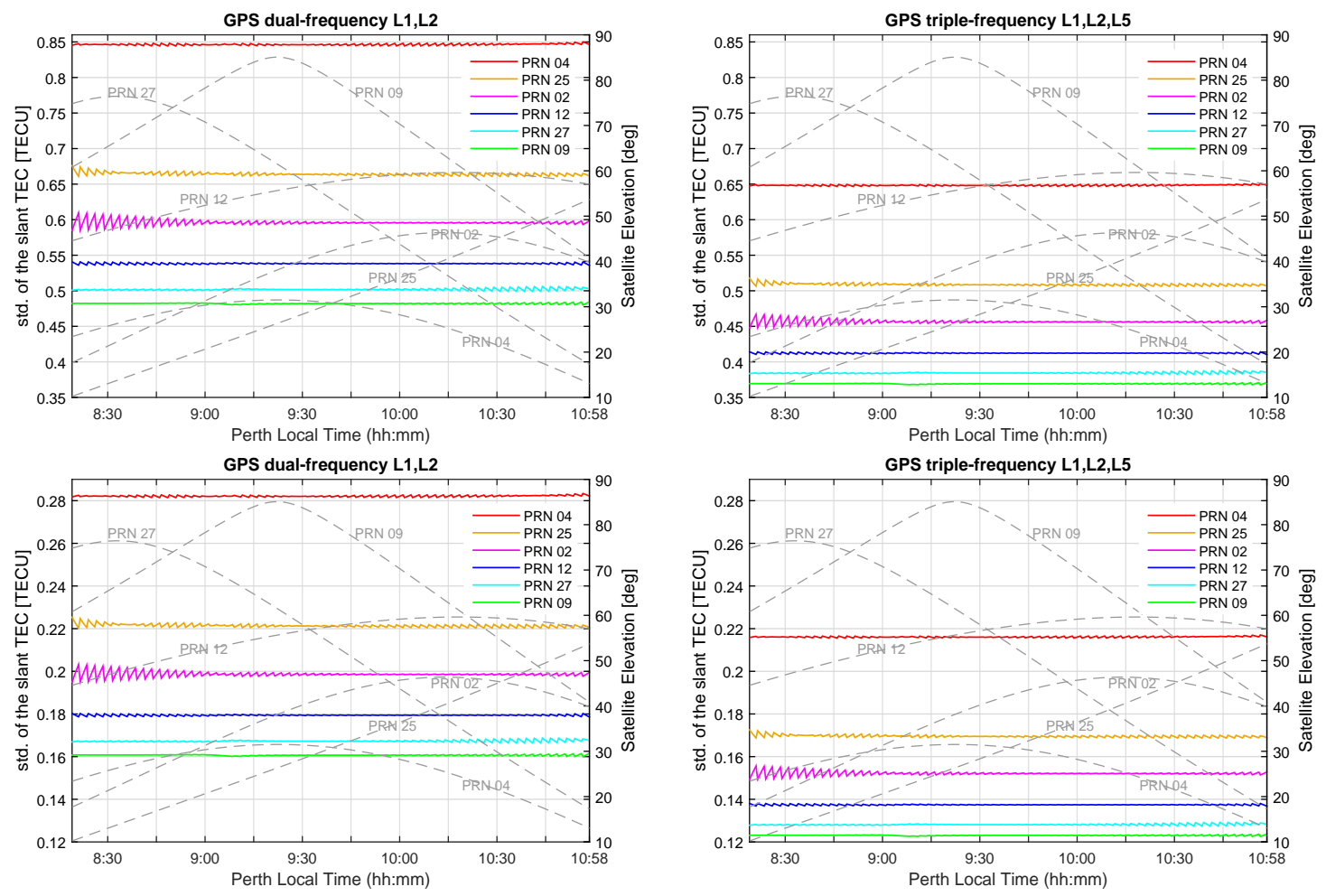

Fig. 7. Standard-deviation [TECU] of the slant TEC $\hat{\boldsymbol{i}}_{r}^{s}$ obtained by the bi-quadratic basis functions (75) for the GPS dual-frequency L1,L2 (Left) and GPS triple-frequency L1,L2,L5 scenarios (Right). Top: single-antenna mode $(n=1)$; Bottom: array-aided mode $(n=9)$. The values follow by setting $\sigma_{p} \approx 1.85$ [TECU], $\epsilon=0.0001$, and the refreshing interval $\tau=2.5$ [min]. The satellite elevation $\theta_{t}^{s}$ [deg] is depicted in grey dashed lines, where use is made of the exponential elevation weights $w_{t}^{s}=\left[1+10 \exp \left(-\theta_{t}^{s} / 10\right)\right]^{-2}$ [35]. The observational sampling rate is 30 seconds, thus $k=320$ epochs $=160$ minutes.

where $\alpha$ and $\beta$, respectively, denote the longitude and latitude of the ionospheric piercing point (IPP) of their corresponding satellite/receiver. The mapping function $\mathcal{M}_{r}^{s}$ is computed as [5]

$$
\mathcal{M}_{r}^{s}=\frac{1}{\cos \left(z^{\prime}\right)}, \quad \sin \left(z^{\prime}\right)=\frac{R}{R+H} \sin (z)
$$

with $R$ and $H=450[\mathrm{~km}]$ being the mean Earth's radius and height of the layer, respectively. The angles $z$ and $z^{\prime}$ denote the zenith angles to the satellite through the receiver and its IPP, respectively.

Figure 7 shows the standard deviations of the corresponding slant TEC $\hat{\boldsymbol{i}}_{r}^{s}$ over time for a GPS data-set of 160 minutes. The observational sampling rate is 30 seconds, thus $k=320$ epochs. The zigzag pattern, highlighted in the figure, is attributed to the chosen refreshing interval $\tau=5$ epochs $=2.5$ minutes, thus $K=64$. The largest standard deviations belong to the satellite with the lowest elevation, i.e. PRN04 (red lines), while the smallest ones belong to the satellite with the highest elevation, PRN09 (green lines). This results from the elevation-dependent weight matrices $\boldsymbol{W}_{t}$.

In the top panel of the figure, the results of the single-antenna mode $(n=1)$ are given. For the dual-frequency case (left), the standard deviations range from 0.54 to 0.85 [TECU]. These values, respectively, drop to 0.37 and 0.65 [TECU] for the triple-frequency case (right). In the bottom panel of the figure, the results of the array-aided mode $(n=9)$ are given. In agreement with the analytical expressions (72), the corresponding standard-deviations decreases by a factor of $\sqrt{9}=3$, thereby obeying the 1-over- $\sqrt{n}$ rule. In case of the triple-frequency scenario $(f=3)$, aided by 9 array antennas, the largest standard deviation is about 0.22 [TECU], presenting a significant improvement over its single-antenna dual-frequency version, i.e. 0.85 [TECU].

\section{SUMMARY AND CONCLUSIONS}

In this contribution the multi-frequency estimability and precision analysis of GNSS ionospheric sensing was presented for the first time. A diagram summarizing the main outcomes of the estimability and precision analysis of the GNSS-derived TEC solutions is given in Figure 8 (a). It was demonstrated that choosing different $\mathcal{S}$-bases leads to different ionospheric observables. Two such $\mathcal{S}$-bases were chosen to eliminate the rank-deficiencies in the GNSS model (20), thus resulting in the two different full-rank models (23) and (24). These two models represent two different estimable ionospheric parameters, namely 1) the phase-driven parameters $\boldsymbol{i}_{r ; \phi}$ (cf. 9) and 2) the code-driven parameters $\boldsymbol{i}_{r ; p}$ (cf. 15). Their interpretations are provided in Tables I and II. Through the one-to-one correspondence (Table III), it was demonstrated that the two models contain 


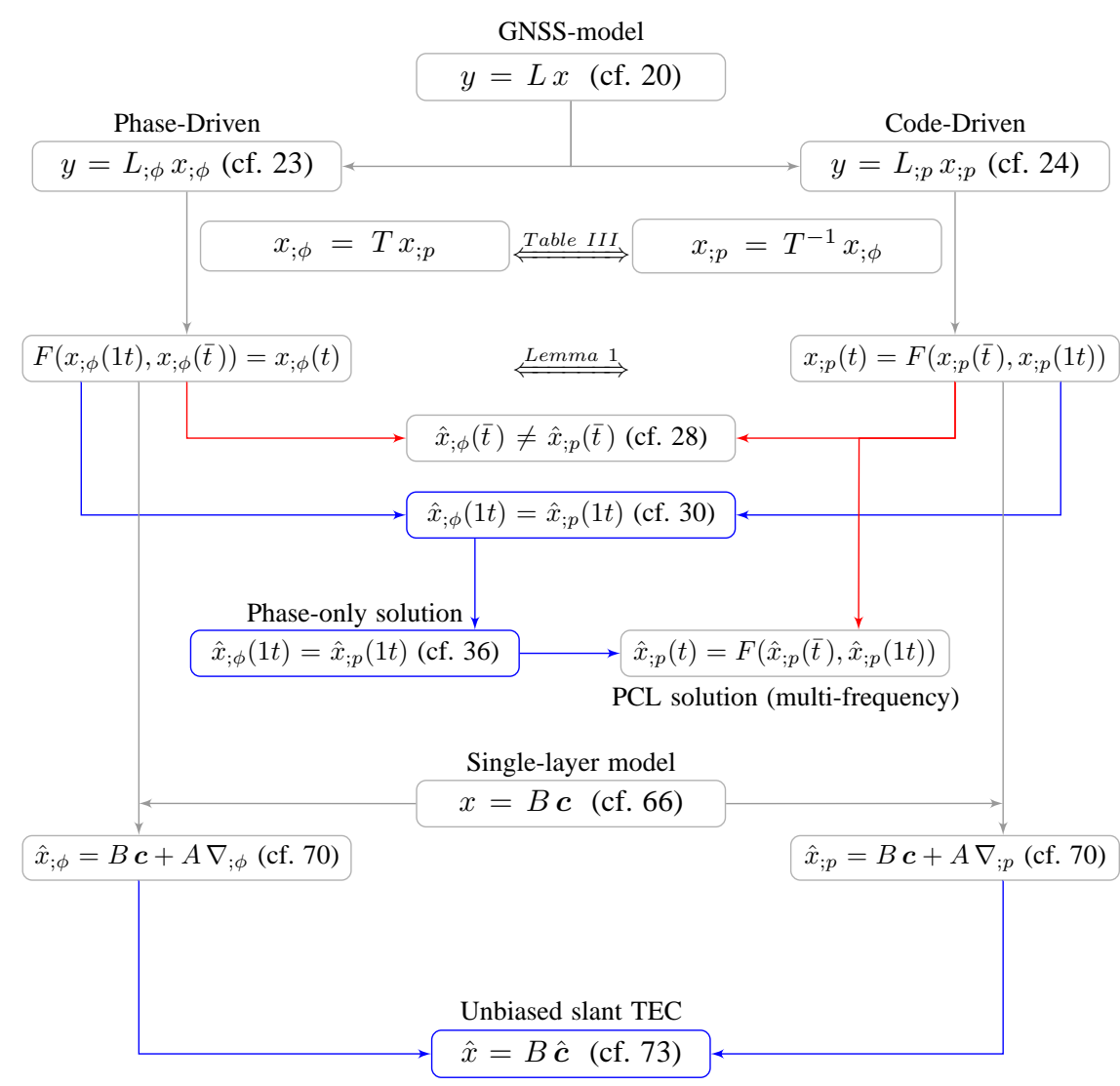

(a)

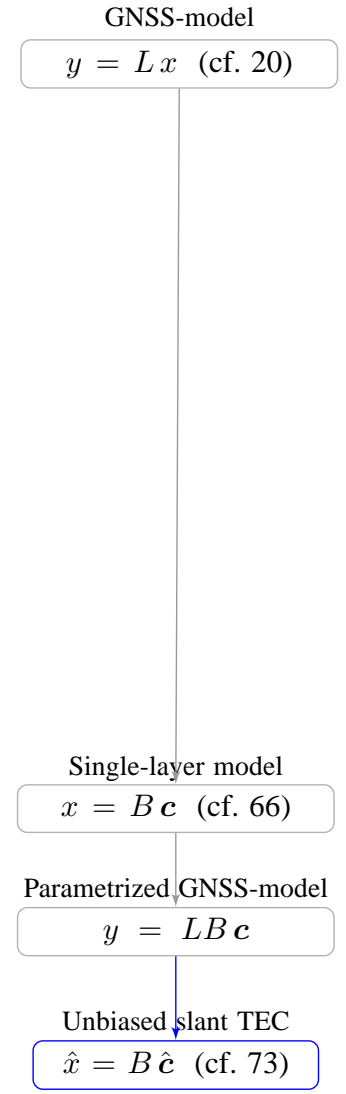

(b)

Fig. 8. (a): Diagram summarizing the main outcomes of the estimability and precision analysis of the GNSS-derived TEC solutions. (b): Parametrizing the GNSS model using the single-layer model and determining the TEC solutions in one go. The contribution of the time-averaged components is highlighted by the red arrows, whereas that of the time-differenced components is depicted by the blue arrows.

the same information. Working with any of the two forms of estimable ionospheric parameters must therefore result in the same TEC outcomes, provided that the TEC-estimation process is based on a properly weighted least-squares adjustment. The TEC-estimation must therefore be based on a rigorous application of the BLUE (best linear unbiased estimation) principle. It is therefore the inverse of the variance matrices $\mathrm{D}\left(\hat{\boldsymbol{i}}_{; \phi}\right)$ and $\mathrm{D}\left(\hat{\boldsymbol{i}}_{; p}\right)$ that need to be taken as weight matrix of the underlying least-squares adjustment. If the weight matrix is not properly chosen, the stated invariance in the TEC determination is not necessarily preserved and outcomes may be obtained that depend on the arbitrarily chosen $\mathcal{S}$-basis.

To help facilitate our precision analysis, the ionospheric observables $\hat{\boldsymbol{i}}_{r ; \phi}$ and $\hat{\boldsymbol{i}}_{r ; p}$ were decomposed into their time-averaged and time-differenced components (Lemma 1), with their single-antenna multi-epoch analytical solution given in Theorem 1. The solutions of their time-differenced components were shown to be identical, i.e. $\hat{\boldsymbol{i}}_{r ; \phi}(1 t)=\hat{\boldsymbol{i}}_{r ; p}(1 t)$. This is in agreement with the fact that the time-differenced components of both the phase- and code-driven ionospheric parameters describe the same quantity, i.e. the time-differences of the unbiased slant ionospheric delays (cf. 34). The solutions of their time-averaged components are different however. The root of this difference lies in the different interpretations assigned to the estimable ionospheric parameters. The phase-driven ionospheric delay $\boldsymbol{i}_{r ; \phi}(\bar{t})$ is biased by the ambiguities (cf. 9), while the code-driven ionospheric delay $\boldsymbol{i}_{r ; p}(\bar{t})$ is biased by the code-biases (cf. 15). It therefore follows that neither their least-squares solution nor their precision are the same. As a consequence, the precision of the phase-driven observables $\hat{\boldsymbol{i}}_{r ; \phi}$ is governed by the very precise phase data, whereas that of the code-driven observables $\hat{\boldsymbol{i}}_{r ; p}$ is dominated by the rather poor precision of the code data.

We also presented the phase-only solution as a special case. In doing so we generalized the widely-used dual-frequency PCL technique to the multi-frequency case and showed how it fits in our general least-squares framework (Corollary 3 ). As Figure 8 (a) shows, the multi-frequency version of the PCL solution is structured by the phase-only solution of the time-differenced component $\boldsymbol{i}_{r ; p}(1 t)$ and the solution of the code-driven time-averaged component $\boldsymbol{i}_{r ; p}(\bar{t})$. To further improve the precision of the ionospheric observables and therefore the precision of the estimated TEC, we introduced the array-aided concept (cf. Figure 4). While both the phase- and code-driven ionospheric observables benefit from the extra aiding antennas, their responses to array ambiguity resolution are quite distinct. The precision of the code-driven observables $\hat{\boldsymbol{i}}_{r ; p}$ remains unchanged after ambiguity resolution. This is not the case however, with the precision of the phase-driven observables $\hat{\boldsymbol{i}}_{r ; \phi}$. Its time-averaged ambiguity float component does not profit from the data of the aiding antennas. This happens only after successful ambiguity 
resolution has taken place. We further showed that the array-aided time-differenced solutions follow as the 'antenna-averaged' version of their single-antenna counterparts $\hat{\boldsymbol{i}}_{r ; \phi}(1 t)$ and $\hat{\boldsymbol{i}}_{r ; p}(1 t)$ (cf. 57). As it was shown, using the single-layer model as an example, that it is these time-differenced components of the ionospheric observables that govern the least-squares solutions of the slant TEC $\hat{\boldsymbol{i}}_{r}$ (Theorem 3), it is important that one does not base one's precision analysis of TEC on that of the ionospheric observables $\hat{\boldsymbol{i}}_{r ; \phi}(t)$ or $\hat{\boldsymbol{i}}_{r ; p}(t)$.

\section{APPENDIX}

Proof of Theorem 1: We will only prove the solutions of the code-driven parameters $\boldsymbol{i}_{r ; p}(\bar{t})$ and $\boldsymbol{i}_{r ; p}(1 t)$. The proof of the phase-driven parameters $\boldsymbol{i}_{r ; \phi}(\bar{t})$ and $\boldsymbol{i}_{r ; \phi}(1 t)$ goes along similar lines. Let us first consider the time-average solution (28). The time-averaged version of the full-rank observation equations (24) reads

$$
\left[\begin{array}{c}
\phi_{r}(\bar{t}) \\
\boldsymbol{p}_{r}(\bar{t})
\end{array}\right]=\left(\left[\begin{array}{cccc}
-\boldsymbol{\mu}, & \boldsymbol{e}, & 0, & \boldsymbol{I} \\
+\boldsymbol{\mu}, & \boldsymbol{e}, & \boldsymbol{E}, & 0
\end{array}\right] \otimes \boldsymbol{I}_{m}\right)\left[\begin{array}{c}
\boldsymbol{i}_{r ; p}(\bar{t}) \\
\boldsymbol{\rho}_{r ; p}(\bar{t}) \\
\boldsymbol{d}_{r ; p} \\
\boldsymbol{a}_{r ; p}
\end{array}\right]
$$

As this is an invertible system of $2 \mathrm{fm}$ equations in $2 \mathrm{fm}$ unknowns, its solution follows after inversion as

$$
\left[\begin{array}{c}
\hat{\boldsymbol{i}}_{r ; p}(\bar{t}) \\
\hat{\boldsymbol{\rho}}_{r ; p}(\bar{t}) \\
\hat{\boldsymbol{d}}_{r ; p} \\
\hat{\boldsymbol{a}}_{r ; p}
\end{array}\right]=\left(\left[\begin{array}{cc}
0, & \boldsymbol{\mu}_{G F}^{T} \\
0, & \boldsymbol{\mu}_{I F}^{T} \\
0, & \boldsymbol{E}^{-} \\
\boldsymbol{I}, & \boldsymbol{M}
\end{array}\right] \otimes \boldsymbol{I}_{m}\right)\left[\begin{array}{l}
\boldsymbol{\phi}_{r}(\bar{t}) \\
\boldsymbol{p}_{r}(\bar{t})
\end{array}\right]
$$

with $\boldsymbol{M}=\boldsymbol{\mu} \boldsymbol{\mu}_{G F}^{T}-\boldsymbol{e} \boldsymbol{\mu}_{I F}^{T}$. The time-average solution (28) follows from the first set of equations (79). The corresponding variance matrix, given in (29), follows by an application of the error propagation law to (28).

We now turn our attention to the time-differenced solution (30). The time-differenced version of the full-rank observation equations (24) reads

$$
\left[\begin{array}{l}
\phi_{r}(1 t) \\
\boldsymbol{p}_{r}(1 t)
\end{array}\right]=\left(\left[\begin{array}{cc}
-\boldsymbol{\mu}, & \boldsymbol{e} \\
+\boldsymbol{\mu}, & \boldsymbol{e}
\end{array}\right] \otimes \boldsymbol{I}_{m}\right)\left[\begin{array}{c}
\boldsymbol{i}_{r ; p}(1 t) \\
\boldsymbol{\rho}_{r ; p}(1 t)
\end{array}\right], \quad t=2, \ldots, k
$$

One can reduce the above system of equations by eliminating the coefficients of $\boldsymbol{\rho}_{r ; p}(1 t)$. This can be done through premultiplying (80) by the orthogonal-complement basis matrix

$$
\left(\left[\begin{array}{l}
\boldsymbol{e} \\
\boldsymbol{e}
\end{array}\right] \otimes \boldsymbol{I}_{m}\right)^{\perp T}=\left[\begin{array}{cc}
-\boldsymbol{I} & \boldsymbol{I} \\
\boldsymbol{D}_{f}^{T} & \epsilon \boldsymbol{D}_{f}^{T}
\end{array}\right] \otimes \boldsymbol{I}_{m}
$$

which gives the following two uncorrelated sets of observation equations

$$
\begin{array}{lll}
(1): & \boldsymbol{p}_{r}(1 t)-\boldsymbol{\phi}_{r}(1 t) & =-2\left[\boldsymbol{\mu} \otimes \boldsymbol{I}_{m}\right] \boldsymbol{i}_{r ; p}(1 t) \\
(2): & \left(\boldsymbol{D}_{f}^{T} \otimes \boldsymbol{I}_{m}\right)\left[\epsilon \boldsymbol{p}_{r}(1 t)+\boldsymbol{\phi}_{r}(1 t)\right]=-(1-\epsilon)\left[\boldsymbol{D}_{f}^{T} \boldsymbol{\mu} \otimes \boldsymbol{I}_{m}\right] \boldsymbol{i}_{r ; p}(1 t)
\end{array}
$$

Using the observations' variance matrix (22), the corresponding normal matrices $\boldsymbol{U}_{i}$ and the right-hand vectors $\boldsymbol{l}_{i}(i=1,2)$ are obtained as

$$
\begin{array}{ll}
\boldsymbol{U}_{1}=\frac{4\left(\boldsymbol{\mu}^{T} \boldsymbol{\mu}\right)}{(1+\epsilon) \sigma_{p}^{2}}\left(\boldsymbol{W}_{1}^{-1}+\boldsymbol{W}_{t}^{-1}\right)^{-1} ; & \boldsymbol{l}_{1}=-\left[\frac{2 \boldsymbol{\mu}^{T}}{(1+\epsilon) \sigma_{p}^{2}} \otimes\left(\boldsymbol{W}_{1}^{-1}+\boldsymbol{W}_{t}^{-1}\right)^{-1}\right]\left[\boldsymbol{p}_{r}(1 t)-\boldsymbol{\phi}_{r}(1 t)\right] \\
\boldsymbol{U}_{2}=\frac{(1-\epsilon)^{2}\left(\boldsymbol{\mu}^{T} \mathcal{P}_{D_{f}} \boldsymbol{\mu}\right)}{(1+\epsilon) \sigma_{\phi}^{2}}\left(\boldsymbol{W}_{1}^{-1}+\boldsymbol{W}_{t}^{-1}\right)^{-1} ; & \boldsymbol{l}_{2}=-\left[\frac{(1-\epsilon) \boldsymbol{\mu}^{T} \mathcal{P}_{D_{f}}}{(1+\epsilon) \sigma_{\phi}^{2}} \otimes\left(\boldsymbol{W}_{1}^{-1}+\boldsymbol{W}_{t}^{-1}\right)^{-1}\right]\left[\epsilon \boldsymbol{p}_{r}(1 t)+\boldsymbol{\phi}_{r}(1 t)\right]
\end{array}
$$

with $\mathcal{P}_{D_{f}}=\boldsymbol{D}_{f}\left(\boldsymbol{D}_{f}^{T} \boldsymbol{D}_{f}\right)^{-1} \boldsymbol{D}_{f}$. The solution $\hat{\boldsymbol{i}}_{r ; p}(1 t)$ is therefore obtained as

$$
\hat{\boldsymbol{i}}_{r ; p}(1 t)=\left(\boldsymbol{U}_{1}+\boldsymbol{U}_{2}\right)^{-1}\left(\boldsymbol{l}_{1}+\boldsymbol{l}_{2}\right)
$$

The equality (30) follows by substituting

$$
\left(\boldsymbol{U}_{1}+\boldsymbol{U}_{2}\right)^{-1}=\frac{(1+\epsilon) \sigma_{\phi}^{2}}{f \gamma}\left(\boldsymbol{W}_{1}^{-1}+\boldsymbol{W}_{t}^{-1}\right)
$$

into (84), together with the identities

$$
\begin{array}{ll}
\boldsymbol{\mu}^{T} \boldsymbol{\mu} & =f\left(\bar{\mu}^{2}+\sigma_{\mu}^{2}\right) \\
\boldsymbol{\mu}^{T} \mathcal{P}_{D_{f}} \boldsymbol{\mu} & =f \sigma_{\mu}^{2}, \quad \boldsymbol{\mu}^{T} \mathcal{P}_{D_{f}}=(\boldsymbol{\mu}-\bar{\mu} \boldsymbol{e})^{T}
\end{array}
$$

The corresponding (co)variance matrices, given in (31), follow by an application of the error propagation law to (30). 
Proof of Lemma 2: To form the array-aided condition equations, we eliminate the unknown parameters involved in (50). We first start with the time-averaged version (50) as

$$
\begin{aligned}
& \boldsymbol{\Phi}(\bar{t}) \boldsymbol{D}_{n}=\left(\boldsymbol{e} \otimes \boldsymbol{I}_{m}\right) \boldsymbol{\rho}(\bar{t}) \boldsymbol{D}_{n}+\mathcal{A} \boldsymbol{D}_{n} \\
& \boldsymbol{P}(\bar{t}) \boldsymbol{D}_{n}=\left(\boldsymbol{e} \otimes \boldsymbol{I}_{m}\right) \boldsymbol{\rho}(\bar{t}) \boldsymbol{D}_{n}+\mathcal{D} \boldsymbol{D}_{n}
\end{aligned}
$$

The receiver phase and code biases can be eliminated by applying the between-satellite differencing matrix $\boldsymbol{D}_{m}$, that is

$$
\begin{aligned}
\left(\boldsymbol{I} \otimes \boldsymbol{D}_{m}^{T}\right) \boldsymbol{\Phi}(\bar{t}) \boldsymbol{D}_{n} & =\left(\boldsymbol{e} \otimes \boldsymbol{D}_{m}^{T}\right) \boldsymbol{\rho}(\bar{t}) \boldsymbol{D}_{n}+\left(\boldsymbol{\Lambda} \otimes \boldsymbol{I}_{m-1}\right) \boldsymbol{Z} \\
\left(\boldsymbol{I} \otimes \boldsymbol{D}_{m}^{T}\right) \boldsymbol{P}(\bar{t}) \boldsymbol{D}_{n} & =\left(\boldsymbol{e} \otimes \boldsymbol{D}_{m}^{T}\right) \boldsymbol{\rho}(\bar{t}) \boldsymbol{D}_{n}
\end{aligned}
$$

where use is made of the equality (54). To eliminate $\rho(\bar{t})$, we pre-multiply (80) by the orthogonal-complement basis matrix

$$
\left(\left[\begin{array}{c}
\boldsymbol{e} \\
\boldsymbol{e}
\end{array}\right] \otimes \boldsymbol{D}_{m}^{T}\right)^{\perp T}=\left[\begin{array}{cc}
\boldsymbol{D}_{f}^{T} & 0 \\
0 & \boldsymbol{D}_{f}^{T} \\
\boldsymbol{e}^{T} & -\boldsymbol{e}^{T}
\end{array}\right] \otimes \boldsymbol{I}_{m-1},
$$

This gives the following three sets of condition equations

$$
\begin{aligned}
& \left(\boldsymbol{D}_{f}^{T} \otimes \boldsymbol{D}_{m}^{T}\right) \boldsymbol{\Phi}(\bar{t}) \boldsymbol{D}_{n} \quad=\left(\boldsymbol{D}_{f}^{T} \boldsymbol{\Lambda} \otimes \boldsymbol{I}_{m-1}\right) \boldsymbol{Z} \\
& \left(\boldsymbol{D}_{f}^{T} \otimes \boldsymbol{D}_{m}^{T}\right) \boldsymbol{P}(\bar{t}) \boldsymbol{D}_{n} \quad=0 \\
& \left(\boldsymbol{e}^{T} \otimes \boldsymbol{D}_{m}^{T}\right)[\boldsymbol{\Phi}(\bar{t})-\boldsymbol{P}(\bar{t})] \boldsymbol{D}_{n}=\left(\boldsymbol{e}^{T} \boldsymbol{\Lambda} \otimes \boldsymbol{I}_{m-1}\right) \boldsymbol{Z}
\end{aligned}
$$

But the last set of the above equations are uncorrelated with the single-antenna solutions $\hat{\boldsymbol{i}}_{r ; \diamond}(\bar{t})(\diamond=\{\phi, p\})$, since $\boldsymbol{e}^{T} \boldsymbol{\mu}_{G F}=0$. Thus only the first two sets contribute to the solution, presenting the condition equations $(52)$.

To prove the time-differenced condition equations (52), we pre-multiply the time-differenced version of (50), i.e.

$$
\begin{aligned}
& \left(\boldsymbol{I} \otimes \boldsymbol{I}_{m}\right) \boldsymbol{\Phi}(1 t) \boldsymbol{D}_{n}=\left(\boldsymbol{e} \otimes \boldsymbol{I}_{m}\right) \boldsymbol{\rho}(1 t) \boldsymbol{D}_{n} \\
& \left(\boldsymbol{I} \otimes \boldsymbol{I}_{m}\right) \boldsymbol{P}(1 t) \boldsymbol{D}_{n}=\left(\boldsymbol{e} \otimes \boldsymbol{I}_{m}\right) \boldsymbol{\rho}(1 t) \boldsymbol{D}_{n},
\end{aligned}
$$

by the orthogonal-complement basis matrix given in (81). This gives the the condition equations (53).

Proof of Theorem 2: Given the condition equations (52) and (53), we apply the least-squares conditional adjustment to the single-antenna solutions $\hat{\boldsymbol{i}}_{r ; \diamond}(\bar{t})$ and $\hat{\boldsymbol{i}}_{r ; \diamond}(1 t)(\diamond=\{\phi, p\})$. Let $\boldsymbol{x}$ be the to-be-adjusted quantity, given the condition equations $\boldsymbol{g}=0$. The adjusted version of $\boldsymbol{x}$, say $\hat{\boldsymbol{x}}$, is computed as [40]

$$
\hat{\boldsymbol{x}}=\boldsymbol{x}-\mathrm{C}(\boldsymbol{x}, \boldsymbol{g})(\mathrm{D}(\boldsymbol{g}))^{-1} \boldsymbol{g}
$$

Thus if $\boldsymbol{x}$ is uncorrelated with $\boldsymbol{g}$ (i.e. C $(\boldsymbol{x}, \boldsymbol{g})=0$ ), the quantity $\boldsymbol{x}$ is not adjusted i.e. $\hat{\boldsymbol{x}}=\boldsymbol{x}$.

We first consider the time-averaged component. Let $\hat{\boldsymbol{i}}_{r ; p}(\bar{t})=\left(\boldsymbol{\mu}_{G F}^{T} \otimes \boldsymbol{I}_{m}\right) \boldsymbol{p}_{r}(\bar{t})$ take the role of $\boldsymbol{x}$. Since $\boldsymbol{x}$ is uncorrelated with the first set of equations (52), the role of $\boldsymbol{g}$ is taken by the second set only, that is

$$
\boldsymbol{g} \mapsto \operatorname{vec}\left(\left(\boldsymbol{D}_{f}^{T} \otimes \boldsymbol{D}_{m}^{T}\right) \boldsymbol{P}(\bar{t}) \boldsymbol{D}_{n}\right)
$$

where the vec-operator converts the matrix into a vector. Substitution of the equality

$$
\mathrm{C}(\boldsymbol{x}, \boldsymbol{g})(\mathrm{D}(\boldsymbol{g}))^{-1} \boldsymbol{g}=\left(\boldsymbol{\mu}_{G F}^{T} \otimes \mathcal{P}_{e_{m}}^{\perp}\right)\left[\boldsymbol{p}_{r}(\bar{t})-\boldsymbol{p}_{\bar{r}}(\bar{t})\right]
$$

into (92) yields the adjusted solution $\hat{\boldsymbol{x}} \mapsto \hat{\boldsymbol{i}}_{r ; p}^{\mathrm{ARY}}(\bar{t})$ given in (55). The adjusted solution $\hat{\boldsymbol{i}}_{r ; \phi}^{\mathrm{ARY}}(\bar{t})$ is computed in a similar way. The corresponding variance matrices (56) follow by an application of the error propagation law to (55).

To compute the time-differenced component (57), the role of $\boldsymbol{x}$ is taken by $\hat{\boldsymbol{i}}_{r ; p}(1 t)$. The condition equations (53) would in turn take the role of $\boldsymbol{g}$, that is

$$
\boldsymbol{g} \mapsto\left[\begin{array}{c}
\operatorname{vec}\left([\boldsymbol{P}(1 t)-\boldsymbol{\Phi}(1 t)] \boldsymbol{D}_{n}\right) \\
\operatorname{vec}\left(\left(\boldsymbol{D}_{f}^{T} \otimes \boldsymbol{I}_{m}\right)[\epsilon \boldsymbol{P}(1 t)+\boldsymbol{\Phi}(1 t)] \boldsymbol{D}_{n}\right)
\end{array}\right]
$$

Substitution of the equality

$$
\mathrm{C}(\boldsymbol{x}, \boldsymbol{g})(\mathrm{D}(\boldsymbol{g}))^{-1} \boldsymbol{g}=\left[\hat{\boldsymbol{i}}_{r ; p}(1 t)-\hat{\boldsymbol{i}}_{\bar{r} ; p}(1 t)\right]
$$

into (92) yields the adjusted solution $\hat{\boldsymbol{x}} \mapsto \hat{\boldsymbol{i}}_{r ; p}^{\mathrm{ARY}}(1 t)$ given in (57). The corresponding (co)variance matrices (58) follow by an application of the error propagation law to (57). 
Proof of Theorem (3): We first reduce the system of equations (70) by eliminating the coefficients of $\nabla$. This can be done through pre-multiplying (70) by the orthogonal-complement basis matrix

$$
\left(\left[\begin{array}{c}
\boldsymbol{A} \\
\vdots \\
\boldsymbol{A}
\end{array}\right]\right)^{\perp T}=\left[\begin{array}{c}
\boldsymbol{D}_{K}^{T} \otimes \boldsymbol{I}_{m \tau} \\
\boldsymbol{e}_{K}^{T} \otimes \boldsymbol{A}^{\perp T}
\end{array}\right], \quad \text { with } \quad \boldsymbol{A}^{\perp T}=\boldsymbol{D}_{\tau}^{T} \otimes \boldsymbol{I}_{m},
$$

leading to $K$ sets of observation equations

$$
\begin{aligned}
\left(\hat{\boldsymbol{i}}_{r ; \diamond[l]}-\hat{\boldsymbol{i}}_{r ; \diamond[1]}\right) & =\left(\boldsymbol{B}_{[l]}-\boldsymbol{B}_{[1]}\right) \boldsymbol{c}, \quad l=2, \ldots, K \\
\sum_{l=1}^{K}\left(\boldsymbol{A}^{\perp T} \hat{\boldsymbol{i}}_{r ; \diamond[l]}\right) & =\sum_{l=1}^{K}\left(\boldsymbol{A}^{\perp T} \boldsymbol{B}_{[l]}\right) \boldsymbol{c}
\end{aligned}
$$

Using the observations' variance matrix (22), the normal matrix and the right-hand vector of the first $(K-1)$ sets are obtained as

$$
\boldsymbol{N}_{1}=\sum_{l=1}^{K}\left[\left(\boldsymbol{B}_{[l]}-\overline{\boldsymbol{B}}\right)^{T} \boldsymbol{W}_{[l]}\left(\boldsymbol{B}_{[l]}-\overline{\boldsymbol{B}}\right)\right] ; \quad \boldsymbol{y}_{1}=\sum_{l=1}^{K}\left[\left(\boldsymbol{B}_{[l]}-\bar{B}\right)^{T} \boldsymbol{W}_{[l]}\left(\hat{\boldsymbol{i}}_{r ; \diamond[l]}-\overline{\boldsymbol{i}}_{r ; \diamond}\right)\right]
$$

while those of the last set read

$$
\boldsymbol{N}_{2}=K\left(\bar{B}^{T} \mathcal{P}_{A}^{\perp T} W_{[\bar{l}]} \mathcal{P}_{A}^{\perp} \bar{B}\right) ; \quad \boldsymbol{y}_{2}=K\left(\bar{B}^{T} \mathcal{P}_{A}^{\perp T} W_{[\bar{l}]} \mathcal{P}_{A}^{\perp} \overline{\boldsymbol{i}}_{r ; \diamond}\right)
$$

with $\mathcal{P}_{A}^{\perp}=\boldsymbol{I}_{\tau m}-\boldsymbol{A}\left(\boldsymbol{A}^{T} \boldsymbol{W}_{[\bar{l}]} \boldsymbol{A}\right)^{-1} \boldsymbol{A}^{T} \boldsymbol{W}_{[\bar{l}]}$. The diagonal matrix $\boldsymbol{W}_{[l]}$ contains all the submatrices $\boldsymbol{W}_{t}$ within the $l^{t h}$ time interval (65). The average quantities are given as follows

$$
\boldsymbol{W}_{[\bar{l}]}=\frac{1}{K} \sum_{l=1}^{K} \boldsymbol{W}_{[l]}, \quad\left[\overline{\boldsymbol{B}}, \bar{i}_{r ; \diamond}\right]=\frac{1}{K} \boldsymbol{W}_{[\bar{l}]}^{-1} \sum_{l=1}^{K} \boldsymbol{W}_{[l]}\left[\boldsymbol{B}_{[l]}, \hat{\boldsymbol{i}}_{r ; \diamond[l]}\right]
$$

The normal equations (72) follow by summing the above normal matrices and right-hand vectors, respectively, that is

$$
\boldsymbol{N}=\boldsymbol{N}_{1}+\boldsymbol{N}_{2}, \quad \boldsymbol{y}=\boldsymbol{y}_{1}+\boldsymbol{y}_{2}
$$

According to (98), the right-hand side vector $\boldsymbol{y}$ is a linear function of

$$
\begin{aligned}
& \left(\hat{\boldsymbol{i}}_{r ; \diamond[l]}-\hat{\boldsymbol{i}}_{r ; \diamond[1]}\right), \quad l=2, \ldots, K \\
& \boldsymbol{A}^{\perp T} \hat{\boldsymbol{i}}_{r ; \diamond[l]}
\end{aligned}
$$

In order to show that $\boldsymbol{y}$ is a function of the time-differenced components $\hat{\boldsymbol{i}}_{r ; \phi}(1 t)=\hat{\boldsymbol{i}}_{r ; p}(1 t)$, it therefore suffices to prove that the linear combinations (103) are linear functions of $\hat{\boldsymbol{i}}_{r ; \phi}(1 t)=\hat{\boldsymbol{i}}_{r ; p}(1 t)$. This immediately follows from the identities

$$
\begin{array}{llr}
\left(\hat{\boldsymbol{i}}_{r ; \diamond[l]}-\hat{\boldsymbol{i}}_{r ; \diamond[1]}\right) & = & \left\{\hat{\boldsymbol{i}}_{r ; \diamond[l]}-\left[\boldsymbol{e}_{\tau} \otimes \boldsymbol{I}_{m}\right] \hat{\boldsymbol{i}}_{r ; \diamond}(1)\right\}-\left\{\hat{\boldsymbol{i}}_{r ; \diamond[1]}-\left[\boldsymbol{e}_{\tau} \otimes \boldsymbol{I}_{m}\right] \hat{\boldsymbol{i}}_{r ; \diamond}(1)\right\}, \quad l=2, \ldots, K \\
\boldsymbol{A}^{\perp T} \hat{\boldsymbol{i}}_{r ; \diamond[l]} & =\boldsymbol{A}^{\perp T}\left\{\hat{\boldsymbol{i}}_{r ; \diamond[l]}-\left[\boldsymbol{e}_{\tau} \otimes \boldsymbol{I}_{m}\right] \hat{\boldsymbol{i}}_{r ; \diamond}(1)\right\}
\end{array}
$$

since $\boldsymbol{D}_{\tau}^{T} \boldsymbol{e}_{\tau}=0$.

\section{ACKNOWLEDGMENT}

This work was partially funded by the Chinese Academy of Sciences (CAS) and the Royal Netherlands Academy of Arts and Sciences (KNAW) joint research project "Compass, Galileo and GPS for improved ionosphere modelling", and partially funded by the CRC-SI Positioning Program Project 1.19 "Multi-GNSS PPP-RTK Network Processing". The second author is the recipient of an Australian Research Council (ARC) Federation Fellowship (NO. FF0883188). All this support is gratefully acknowledged.

\section{REFERENCES}

[1] E. Sardon, A. Rius, and N. Zarraoa, "Estimation of the transmitter and receiver differential biases and the ionospheric total electron content from Global Positioning System observations," Radio Science, vol. 29, no. 3, pp. 577-586, 1994.

[2] S. Schaer, G. Beutler, L. Mervart, M. Rothacher, and U. Wild, "Global and regional ionosphere models using the GPS double difference phase observable," in Proceedings of the IGS Workshop on Special Topics on New Directions, 77-92 Potsdam, Germany, May 15-27, 1995.

[3] E. Sardon and N. Zarraoa, "Estimation of total electron content using GPS data: How stable are the differential satellite and receiver instrumental biases?" Radio Science, vol. 32, no. 5, pp. 1899-1910, 1997.

[4] A. Mannucci, B. Wilson, D. Yuan, C. Ho, U. Lindqwister, and T. Runge, "A global mapping technique for GPS-derived ionospheric total electron content measurements," Radio Science, vol. 33, no. 3, pp. 565-582, 1998.

[5] S. Schaer, "Mapping and predicting the Earth's ionosphere using the Global Positioning System," Ph.D. dissertation, University of Bern, Bern, Switzerland, 1999. 
[6] M. Hernández-Pajares, J. Zornoza, J. S. Subirana, R. Farnworth, and S. Soley, "EGNOS test bed ionospheric corrections under the October and November 2003 storms," Geoscience and Remote Sensing, IEEE Transactions on, vol. 43, no. 10, pp. 2283-2293, 2005.

[7] J. M. Pallarés, G. Ruffini, and L. Ruffini, "Ionospheric tomography using GNSS reflections," Geoscience and Remote Sensing, IEEE Transactions on, vol. 43, no. 2, pp. 321-326, 2005.

[8] L. Ciraolo, F. Azpilicueta, C. Brunini, A. Meza, and S. Radicella, "Calibration errors on experimental slant total electron content (TEC) determined with GPS," J Geod, vol. 81, no. 2, pp. 111-120, 2007.

[9] C. Brunini and F. J. Azpilicueta, "Accuracy assessment of the GPS-based slant total electron content," J Geod, vol. 83, no. 8, pp. 773-785, 2009.

[10] X. Yue, W. S. Schreiner, Y.-H. Kuo, J. J. Braun, Y.-C. Lin, and W. Wan, "Observing system simulation experiment study on imaging the ionosphere by assimilating observations from ground GNSS, LEO-based radio occultation and ocean reflection, and cross link," Geoscience and Remote Sensing, IEEE Transactions on, vol. 52, no. 7, pp. 3759-3773, 2014.

[11] P. Chen, W. Yao, and X. Zhu, "Combination of ground-and space-based data to establish a global ionospheric grid model," Geoscience and Remote Sensing, IEEE Transactions on, vol. 53, no. 2, pp. 1073-1081, 2015.

[12] P. J. G. Teunissen, "The ionosphere-weighted GPS baseline precision in canonical form," J Geod, vol. 72, no. 2, pp. 107-111, 1998.

[13] D. Odijk, Fast precise GPS positioning in the presence of ionospheric delays. Netherlands, Geodetic Commission, Delft: Ph.D. thesis, Delft University of Technology, Publication on Geodesy, 52, 2002.

[14] D. A. Grejner-Brzezinska, P. Wielgosz, I. Kashani, D. A. Smith, P. S. Spencer, D. S. Robertson, G. L. Mader et al., "An analysis of the effects of different network-based ionosphere estimation models on rover positioning accuracy," J GPS, vol. 3, no. 1-2, pp. 115-131, 2004.

[15] J. Juan, M. Hernández-Pajares, J. Sanz, P. Ramos-Bosch, A. Aragon-Angel, R. Orus, W. Ochieng, S. Feng, M. Jofre, P. Coutinho et al., "Enhanced precise point positioning for GNSS users," Geoscience and Remote Sensing, IEEE Transactions on, vol. 50, no. 10, pp. 4213-4222, 2012.

[16] L. Mervart, C. Rocken, T. Iwabuchi, Z. Lukes, and M. Kanzaki, "Precise Point Positioning with Fast Ambiguity Resolution-Prerequisites, Algorithms and Performance," in Proceedings of ION GNSS, 2013, pp. 1176-1185.

[17] D. Odijk, B. S. Arora, and P. J. G. Teunissen, "Predicting the success rate of long-baseline GPS+Galileo (partial) ambiguity resolution," The Journal of Navigation, vol. 67, no. 3, pp. 385-401, 2014

[18] A. Rovira-Garcia, J. M. Juan, J. Sanz, and G. Gonzalez-Casado, "A worldwide ionospheric model for fast precise point positioning," Geoscience and Remote Sensing, IEEE Transactions on, vol. 53, no. 8, pp. 4596-4604, 2015.

[19] N. Jakowski, A. Wehrenpfennig, S. Heise, C. Reigber, H. Lhr, L. Grunwaldt, and T. K. Meehan, "GPS radio occultation measurements of the ionosphere from CHAMP: Early results," Geophysical Research Letters, vol. 29, no. 10, pp. 95-1-95-4, 2002.

[20] W. Baarda, "S-transformations and Criterion Matrices," Netherlands Geodetic Commission, Publ. on Geodesy, New Series, Vol. 5(1), Delft, Tech. Rep., 1973.

[21] P. J. G. Teunissen, Generalized inverses, adjustment, the datum problem and S-transformations. In: Optimization and Design of Geodetic Networks, EW Grafarend and F Sanso (Eds), Springer, 1985.

[22] A. Mannucci, B. Iijima, U. Lindqwister, X. Pi, L. Sparks, and B. Wilson, "GPS and ionosphere," Review of Radio Science 1996-1999, pp. 625-665, 1999.

[23] C. S. Carrano, A. Anghel, R. A. Quinn, and K. M. Groves, "Kalman filter estimation of plasmaspheric total electron content using GPS," Radio Sci., vol. 44, no. RS0A10, 2009

[24] P. J. G. Teunissen, "The least-squares ambiguity decorrelation adjustment: a method for fast GPS integer ambiguity estimation," $J$ Geod, vol. 70, no. $1-2$, pp. $65-82,1995$.

[25] _ _A-PPP: array-aided precise point positioning with global navigation satellite systems," Signal Processing, IEEE Transactions on, vol. 60, no. 6, pp. 2870-2881, 2012.

[26] H. V. Henderson, F. Pukelsheim, and S. R. Searle, "On the History of the Kronecker Product," Linear and Multilinear Algebra, vol. 14, no. 2, pp. $113-120,1983$

[27] P. J. G. Teunissen and A. Kleusberg, GPS for Geodesy, 2nd ed. Springer Berlin, 1998.

[28] B. Hofmann-Wellenhof, H. Lichtenegger, and E. Wasle, GNSS: Global Navigation Satellite Systems: GPS, Glonass, Galileo, and More. Springer, New York, 2008

[29] S. G. Jin, R. Jin, and D. Li, "Assessment of BeiDou differential code bias variations from multi-GNSS network observations," Annales Geophysicae, vol. 34 , no. 2 , pp. 259-269, 2016

[30] B. Zhang and P. J. G. Teunissen, "Characterization of multi-GNSS between-receiver differential code biases using zero and short baselines," Science Bulletin, vol. 60, no. 21, pp. 1840-1849, 2015.

[31] M. Hernández-Pajares, J. M. Juan, J. Sanz, À. Aragón-Àngel, A. García-Rigo, D. Salazar, and M. Escudero, "The ionosphere: effects, GPS modeling and the benefits for space geodetic techniques," J Geod, vol. 85, no. 12, pp. 887-907, 2011.

[32] G. Blewitt, "An automatic editing algorithm for GPS data," Geophysical Research Letters, vol. 17, no. 3, pp. 199-202, 1990.

[33] A. Khodabandeh and P. J. G. Teunissen, "An analytical study of PPP-RTK corrections: precision, correlation and user-impact," J Geod, vol. 89, no. 11, pp. 1109-1132, 2015.

[34] M. Hernández-Pajares, J. M. Juan, J. Sanz, and O. L. Colombo, "Application of ionospheric tomography to real-time GPS carrier-phase ambiguities resolution, at scales of 400-1000 km and with high geomagnetic activity," Geophysical Research Letters, vol. 27, no. 13, pp. 2009-2012, 2000.

[35] H. J. Eueler and C. C. Goad, "On optimal filtering of GPS dual frequency observations without using orbit information," Bulletin godsique, vol. 65, no. 2, pp. 130-143, 1991

[36] B. Li and P. J. G. Teunissen, "GNSS antenna array-aided CORS ambiguity resolution,” J Geod, vol. 88, no. 4, pp. 363-376, 2013.

[37] A. Khodabandeh and P. J. G. Teunissen, "Array-based satellite phase bias sensing: theory and GPS/BeiDou/QZSS results," Meas. Sci. Technol., vol. 25, 2014, 095801.

[38] - "Single-Epoch GNSS Array Integrity: an Analytical Study," IAG Symp, vol. 142, 2016, accepted for publication.

[39] P. J. G. Teunissen, "GPS double difference statistics: with and without using satellite geometry," J Geod, vol. 71, no. 3, pp. 137-148, 1997.

[40] - Adjustment Theory: an Introduction. Delft University Press, 2000, series on Mathematical Geodesy and Positioning.

[41] M. Abdel-salam and Y. Gao, "Precise GPS atmosphere sensing based on un-differenced observations," in Proceedings of ION GPS-2004, Long Beach, CA, 2004, pp. 933-940.

[42] S. Banville and R. B. Langley, "Defining the basis of an integer-levelling procedure for estimating slant total electron content," in Proceedings of ION GNSS 2011, Portland, OR, 2011, pp. 2542-2551.

[43] A. Komjathy, L. Sparks, B. D. Wilson, and A. J. Mannucci, "Automated daily processing of more than 1000 ground-based GPS receivers for studying intense ionospheric storms," Radio Science, vol. 40, no. 6, p. RS6006, 2005.

[44] F. Azpilicueta, C. Brunini, and S. Radicella, "Global ionospheric maps from GPS observations using modip latitude," Advances in Space Research, vol. 38, no. 11, pp. 2324-2331, 2006.

[45] C. Brunini and F. Azpilicueta, "GPS slant total electron content accuracy using the single layer model under different geomagnetic regions and ionospheric conditions," J Geod, vol. 84, no. 5, pp. 293-304, 2010.

[46] S. Bassiri and G. A. Hajj, "Higher-order ionospheric effects on the Global Positioning System observables and means of modeling them," Manuscripta Geodaetica, vol. 13, pp. 280-289, 1993. 